\title{
Theory of Structural Response to Macroscopic Electric Fields in Ferroelectric Systems
}

\author{
Na Sai, Karin M. Rabe and David Vanderbilt \\ Department of Physics and Astronomy, Rutgers University, Piscataway, NJ 08854-8019
}

(Dated: May 20, 2002)

\begin{abstract}
We have developed and implemented a formalism for computing the structural response of a periodic insulating system to a homogeneous static electric field within density-functional perturbation theory (DFPT). We consider the thermodynamic potentials $E(\mathbf{R}, \eta, \mathcal{E})$ and $F(\mathbf{R}, \eta, \mathbf{P})$, whose minimization with respect to the internal structural parameters $\mathbf{R}$ and unit cell strain $\eta$ yields the equilibrium structure at fixed electric field $\mathcal{E}$ and polarization $\mathbf{P}$, respectively. First-order expansion of $E(\mathbf{R}, \eta, \mathcal{E})$ in $\mathcal{E}$ leads to a useful approximation in which $\mathbf{R}(\mathbf{P})$ and $\eta(\mathbf{P})$ can be obtained by simply minimizing the zero-field internal energy with respect to structural coordinates subject to the constraint of a fixed spontaneous polarization $\mathbf{P}$. To facilitate this minimization, we formulate a modified DFPT scheme such that the computed derivatives of the polarization are consistent with the discretized form of the Berry-phase expression. We then describe the application of this approach to several problems associated with bulk and short-period superlattice structures of ferroelectric materials such as $\mathrm{BaTiO}_{3}$ and $\mathrm{PbTiO}_{3}$. These include the effects of compositionally broken inversion symmetry, the equilibrium structure for high values of polarization, field-induced structural phase transitions, and the lattice contributions to the linear and the non-linear dielectric constants.

PACS numbers: PACS numbers: 77.22.Ch, 77.65.Bn, 77.84.Dy, 71.15.-m
\end{abstract}

\section{INTRODUCTION}

As the usefulness of density-functional theory (DFT) for the study of dielectric materials is now well established, one might imagine that calculations of crystalline insulators in the presence of a homogeneous macroscopic electric field should be routine. On the contrary, the presence of an electric field introduces several severe difficulties. 1 . 2 The electric potential acquires a term that is linear in the spatial coordinates, thus violating the periodicity condition underlying Bloch's theorem and acting as a singular perturbation on the electronic eigenstates. Moreover, in principle there is no longer a welldefined ground state for the electrons in a solid in a macroscopic electric field because the energy of the system can be always lowered by transferring electrons from the valence band in one spatial region to the conduction band in a distant region.

One way around these difficulties is to make of density-functional perturbation theory (DFPT), 3. which provides a framework for calculating the perturbative response to infinitesimal electric fields (as well as to atomic displacements and strains). DFPT has been widely adopted for many studies of the dielectric and piezoelectric properties and dynamic effective charges of dielectric materials. However, being a perturbative approach, the method is not capable of treating a finite electric field directly.

A more direct attack on the finite-field problem was made by Nunes and Vanderbilt, 1 who showed that a realspace Wannier-function representation could be used to represent the electronic system in the presence of a finite electric field. In this approach, one minimizes a total-energy functional of a set of field-dependent Wannier functions for a periodic system at fixed electric field. Alternatively, the minimization can also be performed at fixed polarization via a Legendre transformation of the energy functional with the electric field treated as a Lagrange multiplier. The approach was implemented in the DFT context by Fernandez et al. 6 but proved too cumbersome to find widespread utility.

In the present paper we propose a new scheme for the treatment of a dielectric system in a static homogeneous electric field. Our scheme is based on a low-order truncation of the DFPT perturbative expansion in electric field, and the use of this truncated expansion to extrapolate to finite electric field. A key feature of our approach is that, while we keep only low orders in the expansion in electric field, we effectively keep all orders of expansion in the structural degrees of freedom. We demonstrate that even a first-order truncation of the electric-field perturbation provides a very useful and practical scheme. In this context the electric field simply couples to the zerofield polarization, so that the latter plays a central role in our formulation. In fact, it is rather natural to formulate our approach in terms of a constrained minimization procedure in which the DFT energy functional is minimized over all structural degrees of freedom subject to a constraint on the value of the polarization. This allows a two-step approach in which one first maps out the energy surface as a function of polarization in the DFT framework, and then uses this energy surface, augmented by the coupling to the electric field, to obtain the ground-state structure in the presence of the field. We will show that essentially no additional approximations are needed beyond the first-order truncation of the freeenergy expansion in electric field. We will also show that the methodology can be extended to second order (or, in fact, to any desired order) in the electric field.

Before proceeding, we should acknowledge an additional theoretical subtlety associated with the correct choice of exchange-correlation functional in the electric- 
field problem. Gonze, Ghosez and Godby (GGG) argued that the exact Kohn-Sham exchange-correlation energy functional should have a dependence on the macroscopic polarization and formulated a "densitypolarization functional theory" in which there is generally an exchange-correlation contribution to the KohnSham electric field. While this was an important formal development that subsequently received much attention 8.010 it has not yet led to an improved practical exchange-correlation functional. We thus restrict ourselves here to the usual LDA exchange-correlation functional where, because of the locality of the central approximation, the subtleties identified by GGG do not arise.

This paper is organized as follows. In the next section, we present our formalism for computing the structural response of an insulating system to an electric field. Some details of the implementation are presented in Sec. III, including details of our minimization procedure, a discussion of modifications that we made to the DFPT procedure to achieve compatibility with the discretized Berryphase polarization formula, and a description of the technical details of the $a b$-initio pseudopotential calculations. Then, in Sec. IV, we present several sample applications of our method. In Sec. IVA, we show that it provides an alternative approach to the study of short-period ferroelectric syperlattice structures with broken inversion symmetry.11 In Sec. IV B we present a study of the dependence of the internal structural parameters of $\mathrm{BaTiO}_{3}$ on polarization. In Sec. IV $\mathrm{IV}$ we show that our method provides a straightforward way of computing the dielectric susceptibilities and piezoelectric coefficients as functions of the electric field, thus allowing an estimation of the non-linear dielectric and piezoelectric response in a ferroelectric system. Finally, in Sec. IVD, we consider a case in which a full three-dimensional treatment of the polarization and the structural distortions is needed. Specifically, we model the polarization rotation and structural phase transitions induced by the application of a macroscopic electric field to a model ferroelectric system and relate our results to recent experiments in PZN-PT. 12 Finally in Sec. V, we summarize our work and discuss the prospects for future applications of our approach.

\section{FORMALISM}

Our goal is to investigate the effect of a homogeneous static electric field on the structure and polarization of polar insulators, including systems with a nonzero spontaneous polarization. In addition to an efficient approach for computation, we also aim towards a formulation that readily allows an intuitive understanding of the effects of the field. As will become clear below, this will lead us to a formulation in which the polarization plays an especially prominent role.

\section{A. Case of a single minimum}

Let $\mathcal{E}$ be the macroscopic electric field, $\mathbf{R}$ the atomic coordinates, and $\eta$ the lattice strain, and assume that the total energy per unit cell $E(\mathbf{R}, \eta, \mathcal{E})$ has a single local minimum of interest in the $(\mathbf{R}, \eta)$ space for given $\mathcal{E}$. (This restriction is normally appropriate for a paraelectric material, but not for a ferroelectric one. The existence of multiple local minima in the latter case calls for a more careful discussion, which is deferred to the following subsection.) We let

$$
E(\mathcal{E})=\min _{\mathbf{R}, \eta} E(\mathbf{R}, \eta, \mathcal{E})
$$

and denote the location of the minimum by $\mathbf{R}_{\text {eq }}(\mathcal{E})$ and $\eta_{\mathrm{eq}}(\mathcal{E})$. The polarization $\mathbf{P}(\mathbf{R}, \eta, \mathcal{E})$, the thermodynamic conjugate of $\mathcal{E}$, can then be obtained from the expression $\mathbf{P}(\mathbf{R}, \eta, \mathcal{E})=-[d E(\mathbf{R}, \eta, \mathcal{E}) / d \mathcal{E}]_{\mathbf{R}, \eta}$ and $\mathbf{P}(\mathcal{E})$ obtained by evaluating $\mathbf{P}\left(\mathbf{R}_{\text {eq }}(\mathcal{E}), \eta_{\text {eq }}(\mathcal{E}), \mathcal{E}\right)$, or equivalently, $-d E(\mathcal{E}) / d \mathcal{E}$.

We can recast this minimization into a form in which the polarization is more central. Viewing $E(\mathbf{R}, \eta, \mathcal{E})$ as a thermodynamic potential that minimizes to equilibrium values of $\mathbf{R}$ and $\eta$ at fixed $\mathcal{E}$ leads naturally via a Legendre transformation to a thermodynamic potential $F(\mathbf{R}, \eta, \mathbf{P})$ that minimizes to equilibrium values of $\mathbf{R}$ and $\eta$ at fixed $\mathbf{P}$ :

$$
\begin{aligned}
F(\mathbf{R}, \eta, \mathbf{P}) & =\min _{\lambda}[E(\mathbf{R}, \eta, \lambda)+\lambda \cdot \mathbf{P}] \\
& =E(\mathbf{R}, \eta, \lambda(\mathbf{R}, \eta, \mathbf{P}))+\lambda(\mathbf{R}, \eta, \mathbf{P}) \cdot \mathbf{P}(2)
\end{aligned}
$$

with $\lambda(\mathbf{R}, \eta, \mathbf{P})$ being the value at the minimum. This is equivalent to $\mathbf{P}(\mathbf{R}, \eta, \lambda)=\mathbf{P}$, that is, $\lambda(\mathbf{R}, \eta, \mathbf{P})$ is the value of the macroscopic field necessary to produce polarization $\mathbf{P}$ at given $\mathbf{R}$ and $\eta$.

We then define the function

$$
\begin{aligned}
F(\mathbf{P}) & =\min _{\mathbf{R}, \eta} F(\mathbf{R}, \eta, \mathbf{P}) \\
& =F\left(\mathbf{R}_{\mathrm{eq}}(\mathbf{P}), \eta_{\mathrm{eq}}(\mathbf{P}), \mathbf{P}\right)
\end{aligned}
$$

with $\mathbf{R}_{\mathrm{eq}}(\mathbf{P})$ and $\eta_{\mathrm{eq}}(\mathbf{P})$ being the values at the minimum. These structural parameters $\mathbf{R}_{\mathrm{eq}}(\mathbf{P})$ and $\eta_{\mathrm{eq}}(\mathbf{P})$ are in fact equal to $\mathbf{R}_{\text {eq }}(\mathcal{E})$ and $\eta_{\mathrm{eq}}(\mathcal{E})$, the structural parameters defined by minimizing $E(\mathbf{R}, \eta, \mathcal{E})$ at the corresponding fixed $\mathcal{E}=\lambda\left(\mathbf{R}_{\text {eq }}(\mathbf{P}), \eta_{\mathrm{eq}}(\mathbf{P}), \mathbf{P}\right)$. The polarization at this extremum is, as expected,

$$
\begin{aligned}
\mathbf{P}(\mathbf{R}(\mathcal{E}), \eta(\mathcal{E}), \mathcal{E}) & = \\
\mathbf{P}\left(\mathbf{R}_{\mathrm{eq}}(\mathbf{P}), \eta_{\mathrm{eq}}(\mathbf{P}), \lambda\left(\mathbf{R}_{\mathrm{eq}}(\mathbf{P}), \eta_{\mathrm{eq}}(\mathbf{P}), \mathbf{P}\right)\right) & =\mathbf{P} .
\end{aligned}
$$

Finally, we re-express the original minimization as

$$
E(\mathcal{E})=\min _{\mathbf{P}}[F(\mathbf{P})-\mathcal{E} \cdot \mathbf{P}]
$$

In this expression, the electric field $\mathcal{E}$ appears only in the term $-\mathcal{E} \cdot \mathbf{P}$, and thus the effects of the field can be completely understood by investigating the $\mathcal{E}$-independent free energy landscape $F(\mathbf{P})$. 


\section{B. Case of multiple stationary points}

In many cases of interest, the function $E(\mathbf{R}, \eta, \mathcal{E})$ has several local minima, and the essential physics of the problem is to map out the competition between these minima. For example, in a tetragonal ferroelectric like $\mathrm{PbTiO}_{3}$, there are six degenerate minima of this function at $\mathcal{E}=0$, and the application of a nonzero $\mathcal{E}$ breaks the symmetry and establishes one dominant domain orientation of the polarization. However, it may also be of interest to follow the behavior of the other local minima, corresponding to metastable states, as well as other stationary points of this energy surface. For example, saddle points and local maxima of $E(\mathbf{R}, \eta, \mathcal{E})$ can correspond to stable states for fixed $\mathbf{P}$.

In such cases, it is straightforward to generalize the previous discussion by associating a label $(n)$ with each stationary point of interest. Thus the location of the stationary point is denoted by $\mathbf{R}^{(n)}(\mathcal{E})$ and $\eta^{(n)}(\mathcal{E})$, and $E^{(n)}(\mathcal{E})=E\left(\mathbf{R}^{(n)}(\mathcal{E}), \eta^{(n)}(\mathcal{E}), \mathcal{E}\right)$ is the energy at the stationary point. The arguments of the previous subsection carry over much as before. The discussion following Eq. (3) is modified by noting that the minimization of $F(\mathbf{R}, \eta, \mathbf{P})$ with respect to $\mathbf{R}$ and $\eta$ at fixed $\mathbf{P}$ in Eq. (3) will always be associated with one of the stationary points of $E(\mathbf{R}, \eta, \mathcal{E})$ with respect to $\mathbf{R}$ and $\eta$ at the corresponding fixed $\mathcal{E}$; that is, $\mathbf{R}_{\text {eq }}(\mathbf{P})=\mathbf{R}^{(n)}(\mathcal{E})$ and $\eta_{\mathrm{eq}}(\mathbf{P})=\eta^{(n)}(\mathcal{E})$ for some $n$.

Finally, defining the global minimum $E(\mathcal{E})=$ $\min _{n} E^{(n)}(\mathcal{E})$, it is easy to see that Eq. (5) holds as before.

\section{Truncation of the expansion}

The central quantities appearing in the preceding subsections are the energy $E(\mathbf{R}, \eta, \mathcal{E})$ and the polarization $\mathbf{P}(\mathbf{R}, \eta, \mathcal{E})$ in a given electric field $\mathcal{E}$. Unfortunately, there is as yet no rigorous formulation of DFT for the case of finite non-zero $\mathcal{E}$. However, electric-field derivatives of arbitrary order may be computed by the methods of density-functional perturbation theory. We thus expand in $\mathcal{E}$ around $\mathcal{E}=0$ :

$$
\begin{aligned}
E(\mathbf{R}, \eta, \mathcal{E}) & =E(\mathbf{R}, \eta, \mathcal{E}=0)+\left.\sum_{\alpha} \mathcal{E}_{\alpha} \frac{\partial E(\mathbf{R}, \eta, \mathcal{E})}{\partial \mathcal{E}_{\alpha}}\right|_{\mathcal{E}=0} \\
& +\left.\frac{1}{2} \sum_{\alpha \beta} \mathcal{E}_{\alpha} \mathcal{E}_{\beta} \frac{\partial^{2} E(\mathbf{R}, \eta, \mathcal{E})}{\partial \mathcal{E}_{\alpha} \partial \mathcal{E}_{\beta}}\right|_{\mathcal{E}=0}+\cdots
\end{aligned}
$$

Carried to all orders in $\mathcal{E}$, this expansion is exact. However, for sufficiently small fields we can make the approximation of truncating this sum to define $E_{i}(\mathbf{R}, \eta, \mathcal{E})$ as the sum of the first $i+1$ terms in Eq. (6), and $\mathbf{P}_{i}(\mathbf{R}, \eta, \mathcal{E})$ as $-\left.\left(d E_{i}(\mathbf{R}, \eta, \mathcal{E}) / d \mathcal{E}\right)\right|_{\mathbf{R}, \eta}$. Note that this truncation is only in powers of $\mathcal{E}$, and that the dependence on $\mathbf{R}$ and $\eta$ is preserved to all orders.
For many systems, it is already of interest to consider the simplest case $i=1$, where

$$
\begin{gathered}
E_{1}(\mathbf{R}, \eta, \mathcal{E})=E(\mathbf{R}, \eta, 0)-\mathcal{E} \cdot \mathbf{P}(\mathbf{R}, \eta, 0) \\
\mathbf{P}_{1}(\mathbf{R}, \eta, \mathcal{E})=\mathbf{P}(\mathbf{R}, \eta, 0)
\end{gathered}
$$

At this order, the resulting expression

$$
F(\mathbf{P})=\min _{\mathbf{R}, \eta, \lambda}[E(\mathbf{R}, \eta, 0)+\lambda \cdot(\mathbf{P}-\mathbf{P}(\mathbf{R}, \eta, 0))]
$$

can also be interpreted as one in which $\lambda$ appears simply as a Lagrange multiplier implementing the constraint $\mathbf{P}(\mathbf{R}, \eta, 0)=\mathbf{P}$ in the set of equations that minimize $E(\mathbf{R}, \eta, 0)$ over $\mathbf{R}$ and $\eta$, i.e.,

$$
\begin{aligned}
& \frac{\partial E(\mathbf{R}, \eta, 0)}{\partial R_{i \alpha}}-\sum_{\beta} \frac{\partial P_{\beta}(\mathbf{R}, \eta, 0)}{\partial R_{i \alpha}} \lambda_{\beta}=0 \\
& \frac{\partial E(\mathbf{R}, \eta, 0)}{\partial \eta_{\mu}}-\sum_{\beta} \frac{\partial P_{\beta}(\mathbf{R}, \eta, 0)}{\partial \eta_{\mu}} \lambda_{\beta}=0 \\
& P(\mathbf{R}, \eta, 0)=\mathbf{P} .
\end{aligned}
$$

Details of the calculation are described in the next section, and all of the results reported in the following sections are obtained using the $i=1$ expressions.

Generalization of the formalism to order $i=2$ is provided in Appendix A.

\section{Relation to method of Fu and Cohen}

In the remaining part of this section we discussan earlier approach proposed by $\mathrm{Fu}$ and Cohen (FC) 13 These authors carried out a first-principles investigation of the mechanism of rotation of the polarization in $\mathrm{BaTiO}_{3}$ by an applied electric field. Their approach is similar to our $i=1$ case, but involves an additional approximation which we will describe by expressing their procedure in the notation established in this section.

The first step in their approach is the same as our $i=1$ case: to approximate $E(\mathbf{R}, \eta, \mathcal{E})$ by $E_{1}(\mathbf{R}, \eta, \mathcal{E})$. The next step is to perform a constrained minimization, computing

$$
U(\mathbf{Q})=\min _{\mathbf{Q}(\mathbf{R}, \eta)=\mathbf{Q}} E(\mathbf{R}, \eta, 0)
$$

where the constraint is not on the polarization, but on $\mathbf{Q}(\mathbf{R}, \eta)$ where $\mathbf{Q}(\mathbf{R}, \eta)$ is the Ti displacement relative to the average position of the other atoms in the unit cell. $\mathbf{R}(\mathbf{Q})$ and $\eta(\mathbf{Q})$ will be defined as the values of the atomic coordinates and strain at the minimum.

The equilibrium energy $E_{\mathrm{FC}}(\mathcal{E})$, structure $\mathbf{R}\left(\mathbf{Q}_{\text {min }}\right), \eta\left(\mathbf{Q}_{\text {min }}\right)$ and polarization $\mathbf{P}_{\mathrm{FC}}(\mathcal{E})=$ $\mathbf{P}\left(\mathbf{R}\left(\mathbf{Q}_{\min }\right), \eta\left(\mathbf{Q}_{\min }\right), 0\right)$ are then obtained by the minimization

$$
E_{\mathrm{FC}}(\mathcal{E})=\min _{\mathbf{Q}}\{U(\mathbf{Q})-\mathcal{E} \cdot \mathbf{P}(\mathbf{R}(\mathbf{Q}), \eta(\mathbf{Q}), 0)\}
$$


However, the results will in general not be equal to those obtained with our $i=1$ expression

$$
E(\mathcal{E})=\min _{\mathbf{P}}\left\{\min _{\mathbf{P}(\mathbf{R}, \eta, \mathbf{0})=\mathbf{P}} E(\mathbf{R}, \eta, 0)-\mathcal{E} \cdot \mathbf{P}\right\}
$$

The reason is that

$$
\begin{aligned}
E(\mathcal{E}) & =\min _{\mathbf{R}, \eta}\{E(\mathbf{R}, \eta, 0)-\mathcal{E} \cdot \mathbf{P}(\mathbf{R}, \eta, 0)\} \\
& =\min _{\mathbf{Q}}\left\{\min _{\mathbf{Q}(\mathbf{R}, \eta)=\mathbf{Q}}\{E(\mathbf{R}, \eta, 0)-\mathcal{E} \cdot \mathbf{P}(\mathbf{R}, \eta, 0)\}\right\} \\
& \leq \min _{\mathbf{Q}}\left\{\left.\{E(\mathbf{R}, \eta, 0)-\mathcal{E} \cdot \mathbf{P}(\mathbf{R}, \eta, 0)\}\right|_{\mathbf{R}(\mathbf{Q}), \eta(\mathbf{Q})}\right\} \\
& =E_{\mathrm{FC}}(\mathcal{E}) .
\end{aligned}
$$

The point is that the coordinates $\mathbf{R}(\mathbf{Q})$ and $\eta(\mathbf{Q})$ that minimize $E$ alone at fixed $\mathbf{Q}$ are generally not the same as those that would minimize the combination $(E-\mathcal{E} \cdot \mathbf{P})$ at fixed $\mathbf{Q}$. At nonzero $\mathcal{E}$, equality will only be obtained under very special circumstances, for example, if the surfaces of constant $\mathbf{P}$ in $\mathbf{R}, \eta$ space coincide with the surfaces of constant $\mathbf{Q}$, at least for the relatively low energy structures. More specifically, the polarization should be a function only of the $\mathrm{Ti}$ displacement relative to all other atoms independent of the detailed arrangement of those atoms and of the strain. This is not unreasonable for small enough fields in $\mathrm{BaTiO}_{3}$, in which the soft mode is almost a pure $\mathrm{Ti}$ displacement and is well isolated in energy from other polar modes. However, as we will see in the following discussion, this proportionality is never quite exact even for small fields, and the discrepancies grow rapidly as the fields get larger.

\section{METHODOLOGY}

\section{A. Minimization procedure}

We now describe in detail how the minimization of $E(\mathbf{R}, \eta, 0)$ respecting the constraint $P(\mathbf{R}, \eta, 0)=\mathbf{P}$ is implemented. Eq. (9) shows that this constraint can be imposed by a Lagrange multiplier $\lambda$. Therefore, we need to solve the stationary-value problem described by Eqs. (10).

Suppose we make a trial guess of the initial coordinates $\mathbf{R}_{0}$ and strains $\eta_{0}$ for the desired structure. The energy $E(\mathbf{R}, \eta, 0)$ can be expanded up to second order in $\delta \mathbf{R}=$ $\mathbf{R}-\mathbf{R}_{\mathbf{0}}$ and $\delta \eta=\eta-\eta_{0}$ as

$$
\begin{aligned}
E(\mathbf{R}, \eta, 0) & =E\left(\mathbf{R}_{0}, \eta_{0}, 0\right)+\sum_{i \alpha}\left(-F_{i \alpha}\right) \delta R_{i \alpha} \\
& +\sum_{\mu \nu}\left(-\sigma_{\mu}\right) \delta \eta_{\nu}+\frac{1}{2} \sum_{\alpha \beta, i j} K_{\alpha \beta}^{i j} \delta R_{i \alpha} \delta R_{j \beta} \\
& +\frac{1}{2} \sum_{\mu \nu} c_{\mu \nu} \delta \eta_{\mu} \delta \eta_{\nu}+\sum_{i \alpha, \mu} \gamma_{i \alpha}^{\mu} \delta R_{i \alpha} \delta \eta_{\mu}
\end{aligned}
$$

where $F_{i \alpha}$ are the Hellmann-Feynman forces, $\sigma_{\mu}$ are the stresses in Voigt notation, $K_{\alpha \beta}^{i j}$ are the force-constant matrix elements, $c_{\mu \nu}$ are the elastic constants, and $\gamma_{i \alpha \mu}$ are the coupling parameters between the internal coordinates and strains. The corresponding variation in the polarization $\mathbf{P}(\mathbf{R}, \eta, 0)$ is

$\mathbf{P}_{\alpha}(\mathbf{R}, \eta, 0)=\mathbf{P}_{\alpha}\left(\mathbf{R}_{0}, \eta_{0}, 0\right)+\sum_{j \alpha} Z_{\alpha \beta}^{i} \delta R_{i \beta}+\sum_{\mu} e_{\alpha \mu}\left(\mathrm{d} \phi_{\beta}\right)$

where $Z_{\alpha \beta}^{i}=\partial P_{\alpha} / \partial R_{i \beta}$ and $e_{\alpha \mu}=\partial P_{\alpha} / \partial \eta_{\mu}$ are respectively the dynamic effective charge and piezoelectric tensors.

Eqs. (10) lead to the linear system of equations

$$
\left(\begin{array}{ccc}
K & \gamma & Z^{*} \\
\gamma & c & e \\
Z^{*} & e & 0
\end{array}\right)\left(\begin{array}{c}
\delta R \\
\delta \eta \\
\lambda
\end{array}\right)=\left(\begin{array}{c}
F \\
\sigma \\
-\mathcal{P}
\end{array}\right)
$$

for $\delta R, \delta \eta$ and $\lambda$, where $\mathcal{P}$ on the right-hand side denotes the difference between the initial and target values of $\mathbf{P}$. At each step of the minimization, we compute $\delta \mathbf{R}$ and $\delta \eta$, and obtain the new coordinates and strains via $\mathbf{R}_{\mathbf{0}}^{\text {new }}=$ $\mathbf{R}_{0}+\delta \mathbf{R}$ and $\eta_{0}^{\text {new }}=\eta_{0}+\delta \eta$. Then $\mathbf{R}_{\text {new }}$ and $\eta_{\text {new }}$ are chosen as the new "trial" coordinates and strains. This is repeated until convergence is achieved.

For a practical implementation of this procedure we use density-functional perturbation theory, which allows us to compute the coefficients $K_{\alpha \beta}^{i j}$ and $Z_{\alpha \beta}^{i}$ efficiently. The forces $F$ and the stresses $\sigma$ are calculated bythe Hellmann-Feynman theorem with Pulay corrections 14 for the stresses. However, the computation of the remaining quantities in Eq. (17), involving derivatives with respect to strain, requires two additional comments. First, the DFPT calculation of $\gamma, c$ and $e$ is not yet implemented in the current version of the ABINIT package (see Sec. III Q) and the finite-difference calculation of these quantities would be exceedingly tedious. However, we will show that an alternative indirect minimization can be carried out by means of a Devonshire-type Hamiltonian 15 Details will be given in Section IVC and IVD. Second, the most efficient way to compute $\mathcal{P}$ is with a discretized Berry-phase expression. However, the dependence on $\mathbf{R}$ and $\eta$ of the resulting polarization corresponds to the DFPT derivatives exactly only in the limit of a dense kpoint sampling mesh. This issue is discussed and resolved in detail in the next subsection.

Before concluding this subsection, we note that the higher-order formalism can be implemented in an analogous way. However, additional energy derivatives would be needed. The details of the treatment for $i=2$ are presented in Appendix A. For the following study, we will restrict ourselves to the first-order case described by Eq. (\#9).

\section{B. DFPT computation of derivatives of the discretized Berry-phase polarization}

In the implementation of the minimization procedure (Eq. 17), a practical problem arises in connection with 
the calculation of the dynamical effective charges $Z^{*}$ and polarization $\mathbf{P}$. By definition, they should be related by

$$
Z_{\alpha \beta}^{i}=V \frac{\partial P_{\alpha}}{\partial R_{i \beta}},
$$

where $\alpha$ and $\beta$ are Cartesian directions, $i$ is the index for the atom, and $V$ represents the unit cell volume.

However, when the discretized Berry-phase expression is used to compute $\mathbf{P}$ and the DFPT expression is used to compute $Z^{*}$ on the same k-point mesh, Eq. (18) is not satisfied exactly. The discrepancy vanishes in the limit of a dense k-point mesh, but in a practical calculation, which must use a finite mesh, it will result in an inconsistency in the equations for the minimization.

In the Berry-phase theory 16 the polarization is

$$
P_{\alpha}^{\mathrm{BP}}=\frac{i f e}{(2 \pi)^{3}} \int_{\mathrm{BZ}} \sum_{m}^{\mathrm{occ}}\left\langle u_{m \mathbf{k}}\left|\frac{d}{d k_{\alpha}}\right| u_{m \mathbf{k}}\right\rangle d \mathbf{k} .
$$

where $f=2$ for spin degeneracy. $\mathbf{P}^{\mathrm{BP}}$ is computed in practice using a discretized formula which, for the case of isolated bands, takes the form

$$
P_{\alpha}=-\frac{f e}{(2 \pi)^{3}} \int_{A} \mathrm{dk}_{\perp} \sum_{m}^{\mathrm{occ}} \operatorname{Im} \ln \prod_{\mathbf{k} \in \mathcal{S}\left(\mathrm{k}_{\perp}\right)}\left\langle u_{m \mathbf{k}} \mid u_{m, \mathbf{k}+\mathbf{b}}\right\rangle
$$

where the integration over the $2 \mathrm{D} \mathrm{k}_{\perp}$ plane perpendicular to direction $\alpha$ is replaced in practice by a summation over a $2 \mathrm{D}$ mesh. The product runs over a string $\mathcal{S}\left(\mathrm{k}_{\perp}\right)$ of $\mathbf{k}$ points running parallel to direction $\alpha$ at a given $\mathrm{k}_{\perp}$. $\mathbf{b}$ is the separation between neighboring points along the string and $f=2$ is the spin degeneracy factor. The composite-band formulation is presented in Appendix B.

In DFPT 17 there are three equivalent expressions for the $Z^{*}$ tensor. The first is the change in the polarization due to the first-order change in the wavefunctions resulting from an atomic displacement:

$$
Z_{\alpha \beta}^{i}=\frac{i f e V}{(2 \pi)^{3}} \int_{\mathrm{BZ}} \sum_{m}^{\mathrm{occ}}\left\langle\frac{\partial u_{m \mathbf{k}}}{\partial R_{i \alpha}} \mid \frac{\partial u_{m \mathbf{k}}}{\partial k_{\beta}}\right\rangle d \mathbf{k},
$$

where $\partial u_{m \mathbf{k}} / \partial R_{i \alpha}$ is the first-order change of the wavefunction due to the perturbation by displacing an atom belonging to the $i$ th sublattice along the $\alpha$ axis. Alternatively, $\mathrm{Z}^{*}$ can be computed as the derivative of the force along direction $\alpha$ on an atom in the $i$ th sublattice with respect to an electric field along direction $\beta$,

$$
\begin{aligned}
Z_{\alpha \beta}^{i} & =2\left[\frac{V}{(2 \pi)^{3}} \int_{\mathrm{BZ}} \sum_{m}^{\mathrm{occ}} f\left\langle u_{m \mathbf{k}}\left|\frac{\partial v_{\mathrm{ext}}}{\partial R_{i \alpha}}\right| \frac{\partial u_{m \mathbf{k}}}{\partial \mathcal{E}_{\beta}}\right\rangle d \mathbf{k}\right. \\
& \left.+\frac{1}{2} \int_{V} \frac{\partial v_{\mathrm{xc}}(\mathbf{r})}{\partial R_{i \alpha}} \frac{\partial n(\mathbf{r})}{\partial \mathcal{E}_{\beta}} d \mathbf{r}\right],
\end{aligned}
$$

where $\partial u_{m \mathbf{k}} / \partial \mathcal{E}_{\beta}$ is the first-order change of the wavefunctions due to the electric field, and $\partial v_{\text {ext }} / \partial R_{i \alpha}$ and $\partial v_{\mathrm{xc}}(\mathbf{r}) / \partial R_{i \alpha}$ are, respectively, the first-order derivatives of the external potential and the exchange-correlation potential with respect to a $\mathbf{q}=0$ displacement 18 The third expression (omitted here) includes both types of first-order wavefunction changes, and is stationary with respect to small errors in the first-order wavefunctions.

In DFPT, first-order changes $\left|\psi^{(1)}\right\rangle$ in the wavefunctions with respect to a perturbation can be computed as self-consistent solutions of the first-order Sternheimer equations

$$
P_{c}\left(H-\epsilon_{m}\right) P_{c}\left|\psi_{m}^{(1)}\right\rangle=-P_{c} H^{(1)}\left|\psi_{m}^{(0)}\right\rangle
$$

subject to a "parallel transport" gauge constraint目

$$
\left\langle\psi_{n}^{(0)} \mid \psi_{m}^{(1)}\right\rangle=0,
$$

where $H^{(1)}$ is the first-order change in $H$ and $P_{c}$ is the projection operator onto the subspace of the conduction bands, and $n$ and $m$ run only over the valence bands.

In the case of the electric-field perturbation with field in Cartesian direction $\alpha$, the Sternheimer equation takes the form 3

$$
P_{c}\left(H-\epsilon_{m \mathbf{k}}\right) P_{c}\left|\frac{\partial u_{m \mathbf{k}}}{\partial \mathcal{E}_{\alpha}}\right\rangle=-P_{c} H^{(1)}\left|u_{m \mathbf{k}}\right\rangle .
$$

where

$$
H^{(1)}=-i \frac{\partial}{\partial k_{\alpha}}+\frac{d v_{\mathrm{H}}}{d \mathcal{E}_{\alpha}}+\frac{d v_{\mathrm{xc}}(\mathbf{r})}{d \mathcal{E}_{\alpha}} .
$$

As input to this equation, we need the quantity $\partial u_{m \mathbf{k}} / \partial k_{\alpha}$ appearing on the right-hand side. This is obtained by solving a second Sternheimer equation

$$
P_{c}\left(H-\epsilon_{m \mathbf{k}}\right) P_{c}\left|\frac{\partial u_{m \mathbf{k}}}{\partial k_{\alpha}}\right\rangle=-P_{c}\left(\frac{\partial H_{\mathbf{k}}}{\partial k_{\alpha}}\right)\left|u_{m \mathbf{k}}\right\rangle,
$$

where $H_{\mathbf{k}}=\frac{1}{2}(-i \nabla+\mathbf{k})^{2}+v_{\mathrm{KS}}$ and thus $\partial H_{\mathbf{k}} / \partial k_{\alpha}=$ $-i \nabla_{\alpha}+k_{\alpha}$. The presence of the operator $i \partial / \partial k_{\alpha}$ in Eq. (26) is a unique feature that arises from the coupling $\mathcal{E} \cdot \mathbf{P}$ between the macroscopic electric field and the polarization.

Now we come to the main point of this subsection, which is that the derivatives of $\mathbf{P}$ calculated from DFPT are not exactly the derivatives of the discretized Berryphase expression for $\mathbf{P}$ in practical calculations. In particular, for a given $\mathbf{k}$-point sampling, the $Z^{*}$ computed by solving for $\partial\left|u_{m \mathbf{k}}\right\rangle\left\langle\partial k_{\alpha}\right.$ from Eq. (27), and then computing $Z^{*}$ via Eq. (21) or via Eqs. (25) and (22), is not exactly equal to the $Z^{*}$ computed from finite differences of the Berry-phase polarization in Eq. (20). The numerical discrepancy between the Berry-phase polarization and the $Z^{*}$ computed from the DFPT affects the application of the constrained minimization scheme proposed in Section III A because it introduces an inconsistency into the linear system in Eq. (17).

Our cure for this problem is to modify the algorithm by which $\partial\left|u_{m \mathbf{k}}\right\rangle / \partial k_{\alpha}$ is calculated within the DFPT 
framework. Instead of solving the Sternheimer equation (27), we calculate $\partial\left|u_{m \mathbf{k}}\right\rangle / \partial k_{\alpha}$ from finite differences of the ground state Bloch wavefunctions $\left|u_{m \mathbf{k}}\right\rangle$ between the neighboring $\mathbf{k}$-points along the $\alpha$-direction. This approach corresponds to the "perturbation expansion after discretization" formalism discussed by Nunes and Gonze in Ref. 2.

We illustrate our approach here for the case of a single band in one dimension. The generalization to the three-dimensional multi-band case is postponed to Appendix B.

For a single band in one dimension, the Berry-phase polarization is

$$
P=-\frac{f e}{2 \pi} \sum_{k} \operatorname{Im} \ln \left\langle u_{k} \mid u_{k+b}\right\rangle
$$

and its first-order variation reflecting a first-order change in the wavefunction $\left|\delta u_{k}\right\rangle$ is

$$
\begin{aligned}
\delta P & =-\frac{f e}{2 \pi} \sum_{k} \operatorname{Im}\left[\frac{\left\langle\delta u_{k} \mid u_{k+b}\right\rangle}{\left\langle u_{k} \mid u_{k+b}\right\rangle}+\frac{\left\langle u_{k} \mid \delta u_{k+b}\right\rangle}{\left\langle u_{k} \mid u_{k+b}\right\rangle}\right] \\
& =-\frac{f e}{2 \pi} \sum_{k} \operatorname{Im}\left[\frac{\left\langle\delta u_{k} \mid u_{k+b}\right\rangle}{\left\langle u_{k} \mid u_{k+b}\right\rangle}-\frac{\left\langle\delta u_{k} \mid u_{k-b}\right\rangle}{\left\langle u_{k} \mid u_{k-b}\right\rangle}\right] \\
& =\frac{f e b}{\pi} \sum_{k} \operatorname{Re}\left\langle\delta u_{k} \mid v_{k}\right\rangle
\end{aligned}
$$

where

$$
\left|v_{k}\right\rangle=\frac{i}{2 b}\left[\frac{\left|u_{k+b}\right\rangle}{\left\langle u_{k} \mid u_{k+b}\right\rangle}-\frac{\left|u_{k-b}\right\rangle}{\left\langle u_{k} \mid u_{k-b}\right\rangle}\right]
$$

is understood to be a finite-difference approximation to $i \partial\left|u_{k}\right\rangle / \partial k$. Note that Eqs. (29-30) are manifestly gaugeindependent in the sense of being independent of the choice of phases for the $\left|u_{k}\right\rangle$.

In the three-dimensional multi-band case, we just need to replace $v_{k}$ of Eq. (30) by its generalization $v_{m \mathbf{k}, \alpha}$ representing $\partial u_{m \mathbf{k}} / \partial k_{\alpha}$ as discussed in Appendix B; $v_{m \mathbf{k}, \alpha}$ is gauge-independent in the more general sense of being invariant with respect to a unitary rotation among occupied bands on neighboring k-points. This $v_{m \mathbf{k}, \alpha}$ can then be substituted for $\partial u_{m \mathbf{k}} / \partial k_{\alpha}$ in Eq. (21) to compute $Z^{*}$. Or equivalently, it can be inserted into Eq. (25) to compute $\partial u_{m \mathbf{k}} / \partial \mathcal{E}_{\alpha}$, which in turn can be substituted into Eq. (22) to compute $Z^{*}$. In either case, we are guaranteed to obtain the same values of $Z^{*}$ as would be derived from a series of finite-difference calculations of polarization vs. atomic displacement using the same k-point set. This is because Eqs. (29-30) are derived directly from the Berry-phase polarization expression of Eq. (19) using the same $\mathbf{k}$ mesh. Moreover, because the Berry-phase polarization (including ionic contributions) is independent of origin, it also follows that the acoustic sum rule $20 \sum_{i} Z_{\alpha \beta}^{i}=\delta_{\alpha \beta}$ on the components of the dynamic effective charges will be satisfied exactly, which is not the case in conventional linear-response calculations of $Z^{*}$.

\section{Computational Details}

We carried outall the $a b$ initio calculations using the ABINIT package 21 in which we have implemented the above algorithm. ABINIT uses a plane-wave basis and provides multiple norm-conserving $(\mathrm{NC})$ and extended NC pseudopotentials. The discretized formula for the wavefunction derivatives with respect to the wavevectors, Eq. (B14), is introduced in a new subroutine dudk.f, a key ingredient that allows us to carry out the constrainedpolarization minimization scheme.

In Sec. IV A, in order to construct the pseudopotentials for the virtual atoms 11 that enter the heterovalent system $\mathrm{Ba}(\mathrm{Ti}-\delta, \mathrm{Ti}, \mathrm{Ti}+\delta) \mathrm{O}_{3}$, we utilize the $\mathrm{FHI}$ atomic code 22 that generates Troullier-Martin separable normconserving pseudopotentials.23 However, the FHI pseudopotential generation scheme only allows one projector within each angular momentum channel, thus preventing the inclusion of the $3 s$ and $3 p$ states, in addition to $3 d$ and $4 s$ states, in the valence for the Ti pseudopotential. (The same problem occurs for the $5 s$ and $5 p$ states for the $\mathrm{Ba}$ atom.) We generate the pseudopotential in ionized configurations $3 s^{2} 3 p^{6} 3 d^{2} 4 s^{0}$ for Ti and $5 s^{2} 5 p^{6} 6 s^{0}$ for Ba. We used the exchange-correlation energy functional in the Ceperley-Alder 24 form with Perdew and Wang25 parameterization.

The studies described in Sections IVB to IVD have been performed with the highly transferable extendednorm-conserving pseudopotentials proposed by Teter.26 A Perdew-Zunger27 parameterized CeperleyAlder exchange-correlation functional was used. These pseudopotentials include the $\mathrm{Pb} 5 d, 6 s$ and $6 p$, the $\mathrm{Ba}$ $5 s, 5 p$ and $6 s$, the Ti $3 s, 3 p, 4 d$ and $4 s$, and the $\mathrm{O} 2 s$ and $2 p$ electrons in the valence states.

We have used an energy cutoff of 35 Ha throughout. The integrals over the Brillouin zone have been replaced by a sum over a $4 \times 4 \times 4 \mathbf{k}$-point mesh. Both the $\mathbf{k}$ point sampling and the energy cutoff have been tested for good convergence of the phonon eigenvalue and eigenvector properties. We use the same $\mathbf{k}$-point mesh for the Berry-phase calculations. Convergence of the relaxations requires the Hellmann-Feynman forces to be less than $0.02 \mathrm{eV} / \AA$. (In the constrained minimization procedure described by Eq. (17), the forces that are tested for convergence are the ones after projection onto the constant-P subspace.)

\section{SAMPLE APPLICATIONS}

In this section, we illustrate the theory within the firstorder $(i=1)$ formalism (see Section II) by applying it to a series of problems involving ferroelectric, dielectric and piezoelectric properties. In particular, we emphasize that the main purpose of these calculations is to exhibit and understand the nonlinearity in the structural response of the ferroelectric systems to an electric field. Such studies have not previously been widely pursued. We have used 


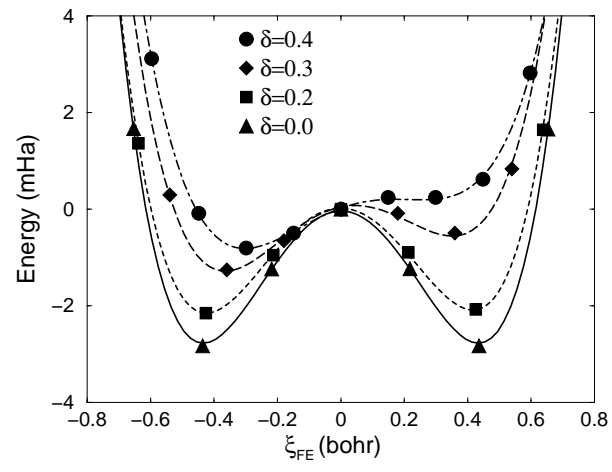

FIG. 1: Energy vs. displacement along the line connecting the two energy minima in the $\mathrm{Ba}(\mathrm{Ti}-\delta, \mathrm{Ti}, \mathrm{Ti}+\delta) \mathrm{O}_{3}$ system, plotted for several values of $\delta$.

$\mathrm{BaTiO}_{3}, \mathrm{PbTiO}_{3}$ and a short-period superlattice structure as our example systems.

\section{A. Inversion symmetry-breaking system}

In a conventional $\mathrm{ABO}_{3}$ perovskite such as $\mathrm{BaTiO}_{3}$, the cubic symmetry of the high-temperature paraelectric phase is spontaneously broken at the transition to the ferroelectric phase. The atomic displacements that occur in the ferroelectric phase give rise to an associated lattice strain, and the ferroelectric state is characterized by a switchable polarization because of the occurrence of degenerate energy minima that are connected by the broken symmetry operations.

Recently, using DFF total-energy methods, we (Sai, Meyer and Vanderbilt 11 ) studied a new class of cubic perovskite compounds in which the composition is modulated in a cyclic sequence of three layers on the $\mathrm{A}$ site (i.e., $\left(A A^{\prime} A^{\prime \prime}\right) B O_{3}$ structures) or on the $\mathrm{B}$ site (i.e., $A\left(B B^{\prime} B^{\prime \prime}\right) O_{3}$ structures). The inversion symmetry that was present in the high-symmetry cubic structure is now permanently broken in these materials by the alternating compositions ordered along the lattice growth direction. This gives rise to important qualitative differences in the energetic behavior of these compounds relative to simple $\mathrm{ABO}_{3}$ perovskites. Most interestingly, it was shown that by using heterovalent compositional substitutions, the strength of the breaking of the inversion symmetry could be tuned through an enormous range, suggesting that such systems could be very promising candidates for new materials with large piezoelectric and other dielectric response properties.11

Such compositionally modulated structures were studied within a model system $\mathrm{Ba}(\mathrm{Ti}-\delta, \mathrm{Ti}, \mathrm{Ti}+\delta) \mathrm{O}_{3}$ where the two atomic species that alternate with $\mathrm{Ti}$ on the $B$ site are virtual atoms that we constructed by varying the nuclear charge of Ti by $\pm \delta$. Therefore, as $\delta$ is tuned continuously from 0 to 1 , we can simulate a set of systems evolving from a conventional $\mathrm{BaTiO}_{3}$ ferroelectric system to a heterovalent $\mathrm{Ba}\left(\mathrm{Sc}_{1 / 3} \mathrm{Ti}_{1 / 3} \mathrm{~V}_{1 / 3}\right) \mathrm{O}_{3}$ one in which all

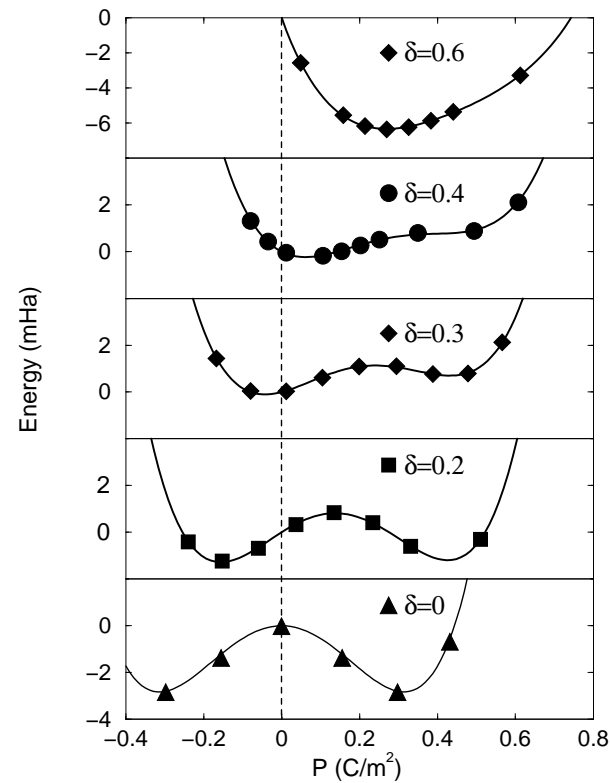

FIG. 2: Energy vs polarization $P$ in $\mathrm{Ba}(\mathrm{Ti}-\delta, \mathrm{Ti}, \mathrm{Ti}+\delta) \mathrm{O}_{3}$ at different $\delta$. Note the saddle point of $E(P)$ shifts in the direction of the secondary (shallower) minimum as $\delta$ increases.

three alternated species are from neighboring columns in the periodic table.

As a consequence of the compositionally broken inversion symmetry, the thermodynamic potential associated with the FE instability does not have the usual symmetric double-well form. Instead, it takes the form of an asymmetric double well, or even of an asymmetric single well, depending on the strength of the compositional perturbation that breaks the symmetry. In normal ferroelectrics, it is sufficient to locate one minimum of the double-well potential; the other is then obviously given by applying the inversion operation. Here, this no longer works. A steepest-descent minimization starting from the ideal structure typically arrives at the primary (deeper) minimum, but the secondary (shallower) minimum can be rather difficult to find in practice.

A procedure was described in Ref. 11 that allows one to search for both minima when they coexist. To illustrate the procedure, we plot in Fig. 1. for several values of $\delta$, the energy as a function of displacement amplitude along the straight line in the 15-dimensional parameter space connecting the primary and secondary minima. (The direction along this line is taken to define the "FE direction" with $\xi_{\mathrm{FE}}=0$ being the midpoint between minima.) Unfortunately, however, it is not possible to plot such a curve for $\delta>0.4$, since only a single minimum exists in this range of $\delta$.

Here, we demonstrate how the current method allows for a much more natural treatment of these systems, especially at large $\delta$. At a fixed value of polarization, we calculate $F(\mathbf{P})$ as in Eq. (9). That is, we minimize the total energy over the internal coordinates subject to the constraint that the spontaneous polarization has a fixed 


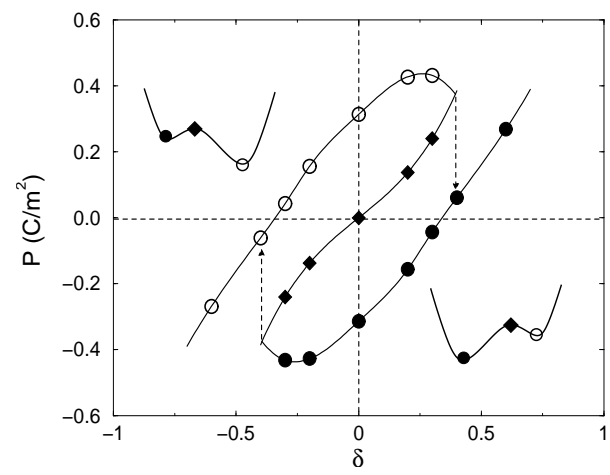

FIG. 3: Calculated polarization at the left minimum (solid circle), right minimum (open circle), and saddle point (diamond) in $\mathrm{Ba}(\mathrm{Ti}-\delta, \mathrm{Ti}, \mathrm{Ti}+\delta) \mathrm{O}_{3}$. Left (right) minimum is the principal one for $\delta>0(\delta<0)$, as shown in insets.

value, following the procedure described in Section III. As in our previous work, this is done in a fixed tetragonal cell.

The energy as a function of the polarization for several values of $\delta$ is illustrated in Fig. 2. We obtain a similar energy evolution as in Fig. 1. However, there are two important qualitative differences. First, the new procedure is not limited to the range of $\delta$ in which both minima exist. At larger $\delta$ (e.g., $\delta=0.6$ ), where the secondary minimum has disappeared due to a strong symmetry-breaking perturbation, the new procedure allows the mapping of the energy to be carried out just as easily as at smaller $\delta$. Second, the horizontal axis of the figure now has a physical meaning of polarization. For example, a glance at Fig. 2 shows an interesting feature, namely that the saddle point is also polarized, unlike in a normal $\mathrm{ABO}_{3}$ compound.

We investigated this interesting feature further by plotting in Fig. 3 the polarization at the saddle point, as well as at the energy minima, as a function of $\delta$. In the "normal" case $\delta=0$, the saddle point is unpolarized and the two equivalent minima carry equal and opposite polarizations. However, all the stationary points are seen to be shifted in the direction of the shallower minimum as $\delta$ is turned on. As a consequence of these shifts, the polarization of the primary minimum changes sign near $\delta \simeq 0.4$. At the critical $\delta$ the saddle point and secondary minimum meet and annihilate. Returning to small values of $\delta$, a closer analysis (not shown) indicates that the polarization at the saddle point increases in proportion to $\delta^{3}$. This observation agrees well with previous studies 11 showing that certain other measures of the effect of the symmetry-breaking perturbation also scale as $\delta^{3}$.

In summary, we have illustrated the convenience and power of our new method in the context of recent work on a new class of ferroelectric materials with compositionally broken inversion symmetry. The new approach is especially useful for studying the case where the compositional perturbation is so strong that only a single local minimum survives. In the former procedure, the

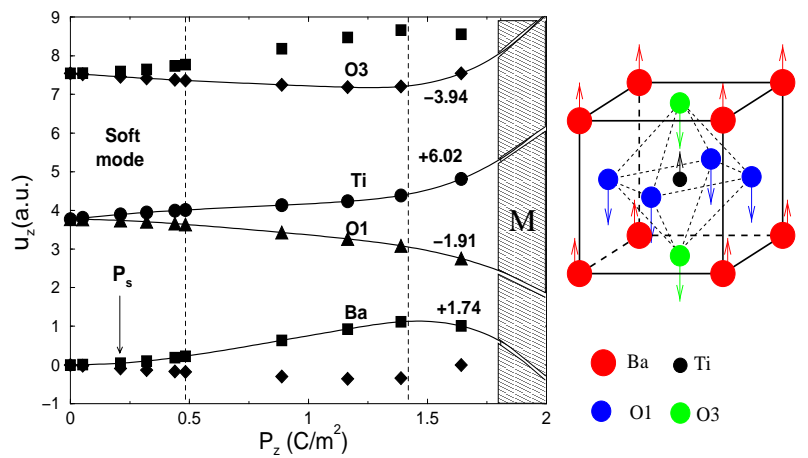

FIG. 4: Fully relaxed $z$ coordinates for each atom in $\mathrm{BaTiO}_{3}$ (see unit cell at right) as a function of polarization $P_{z}$ in the simple-cubic lattice. Curves are cubic-spline fits to calculated points; top- and bottom-most points correspond to translational images of $\mathrm{Ba}$ and $\mathrm{O} 3$ atoms, respectively, in neighboring unit cells. $P_{s}$ marks the spontaneous polarization. The Born effective charge $Z^{*}$ for each atom is marked at $P_{z}=1.64 \mathrm{C} / \mathrm{m}^{2}$. Shaded area indicates metallic regime.

definition of the FE direction was based on the location of two local minima, and was therefore useless when one of the minima had disappeared. On the contrary, in the new method the energy surface can be straightforwardly mapped out as a function of polarization, regardless of whether the secondary minimum exists or not. Moreover, expressing the behavior as a function of polarization provides a much more informative picture of the system. For example, certain interesting and non-trivial behaviors of the polarizations at the saddle points and minima can be elucidated.

\section{B. Structural response in $\mathrm{BaTiO}_{3}$}

In this section, we apply our approach to $\mathrm{BaTiO}_{3}$, one of the most-studied perovskite ferroelectric compounds. It undergoes a sequence of structural phase transitions with decreasing temperature: from the high-temperature cubic to the tetragonal phase at $130^{\circ} \mathrm{C}$, then to an orthorhombic phase at $5^{\circ} \mathrm{C}$ and finally to the ground-state rhombohedral structure at $-90^{\circ} \mathrm{C} .28$ The three successive phases, distortions of the cubic perovskite structure, are characterized by spontaneous polarizations aligned along the [001], [011], and [111] directions respectively.

Here, we focus on the dependence of the internal structural parameters of $\mathrm{BaTiO}_{3}$ on the polarization $\mathbf{P}$. Our calculation is restricted to allow only atomic displacements along the $\hat{z}$ direction in a fixed simple cubic lattice, with full relaxation of the internal structural parameters at fixed polarization $\mathbf{P}=P_{z} \hat{z}$ to yield equilibrium coordinates $\mathbf{R}_{\mathrm{eq}}\left(P_{z}\right)$. In this way, we can investigate the contribution of the internal structural parameters alone, decoupled from the strain degrees of freedom, to the structural response to an electric field, providing a first step towards understanding the nonlinearities of the total structural 


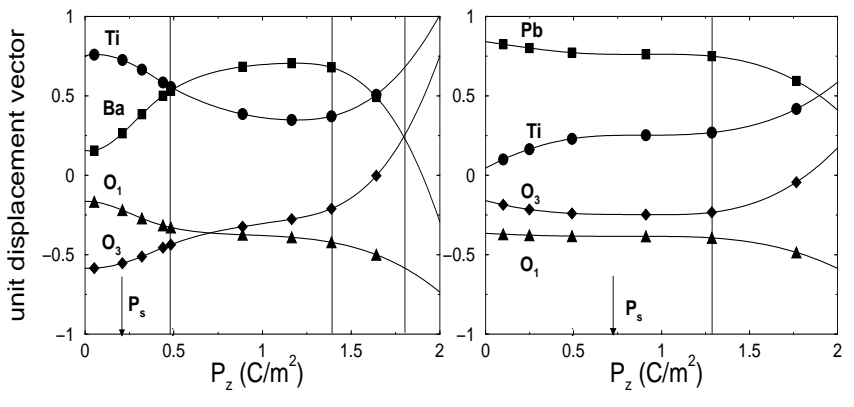

FIG. 5: The component of the unit displacement vector $\xi_{z}$ corresponding to each atom in $\mathrm{BaTiO}_{3}$ (left) and $\mathrm{PbTiO}_{3}$ (right) as a function of polarization $P_{z}$. In the left panel, vertical lines demarcate the regimes of $\mathrm{BaTiO}_{3}$ soft-modelike, $\mathrm{PbTiO}_{3}$ soft-mode-like, atom-pair, and metallic behavior; at right, the single vertical line separates soft-mode-like and atom-pair regimes.

response expected with increasing $\mathcal{E}$.

Experimentally, $\mathrm{BaTiO}_{\beta}$ is known to have a cubic lattice constant of 7.547 a.u.29 Our LDA calculation yields an equilibrium lattice constant of 7.45 a.u., $1.3 \%$ smaller than the experimental value, an error typical of the LDA. As is well known, the ferroelectricinstability depends sensitively on the crystal volume 30.31,32 We therefore choose to work at the experimental cubic lattice constant.

The spontaneous polarization $P_{s}$ is obtained by full relaxation of the internal structural parameters, and is found to be $0.21 \mathrm{C} / \mathrm{m}^{2}$. The relaxed internal coordinates for each $\mathrm{Ba}, \mathrm{Ti}, \mathrm{O} 1$ and $\mathrm{O} 3$ atom are plotted as a function of $P_{z}$ in Fig. 4 . For $P_{z}>P_{s}$, the state can be realized as an equilibrium state in an appropriate fixed electric field, while states with $P_{z}<P_{s}$ are local maxima of $F\left(P_{z}\right)-$ $\mathcal{E} P_{z}$ for some value of $\mathcal{E}$. For example, the value of $\mathcal{E}$ corresponding to $P_{z}=0.48 \mathrm{C} / \mathrm{m}^{2}$ (approximately twice $\left.P_{s}\right)$ is $16 \mathrm{MV} / \mathrm{cm}$.

To focus on the dependence of the character of the distortion on the amplitude $P_{z}$, we define a "unit displacement vector" $\left(\xi_{z}^{\mathrm{Ba}}, \xi_{z}^{\mathrm{Ti}}, \xi_{z}^{\mathrm{O} 1}, \xi_{z}^{\mathrm{O} 3}\right)$ by normalizing the sum of the squared displacements to one. At $P_{z}=P_{s}$, the unit displacement vector is found to be $(0.26,0.73,-0.22,-0.55)$, closely resembling the unstable ferroelectric mode of $\mathrm{cu}-$ bic $\mathrm{BaTiO}_{3}(0.18,0.74,-0.18,-0.59)$ computed from a linear-response calculation. In Fig. 5, we show the $P_{z}$ dependence of the components of the unit displacement vector. If the polarized state were obtained by freezing in a single polar mode, these components would be constant. The actual behavior is considerably more complicated.

Three distinct regimes for the atomic displacement pattern can be clearly observed. For $P_{z}$ below $\approx$ $0.48 \mathrm{C} / \mathrm{m}^{2}$, the relative displacements are similar in character to those of the soft mode. In this regime, the magnitudes of the $\mathrm{Ba}$ and $\mathrm{O} 1$ components increase with $P_{z}$, while the magnitudes of the Ti and O3 components decrease. For $P_{z}$ between roughly $0.48 \mathrm{C} / \mathrm{m}^{2}$ and $1.4 \mathrm{C} / \mathrm{m}^{2}$,

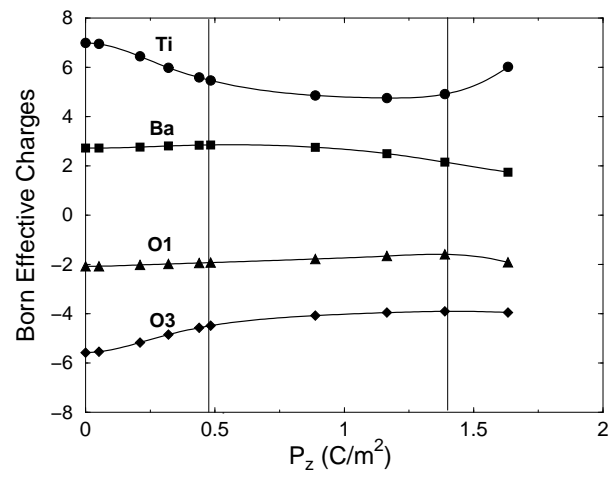

FIG. 6: Computed Born effective charges for each atom in $\mathrm{BaTiO}_{3}$ as a function of the polarization $P_{z}$. For the cubic structure $\left(P_{z}=0\right)$, we obtain $Z_{\mathrm{Ba}}^{*}=2.72, Z_{\mathrm{Ti}}^{*}=6.99, Z_{\mathrm{O}_{\perp}}^{*}=$ $-5.57, Z_{\mathrm{O}_{\|}}^{*}=-2.07$.

the consequence of these opposing trends is that the magnitudes of the $\mathrm{Ba}$ and $\mathrm{O} 1$ displacements actually exceed those of $\mathrm{Ti}$ and $\mathrm{O} 3$, respectively, changing the character of the structural distortion. At $P_{z} \approx 1.4 \mathrm{C} / \mathrm{m}^{2}$, the trend with $P_{z}$ reverses for $\mathrm{Ba}$ and $\mathrm{Ti}$, so that as the polarization increases further, the $\mathrm{Ba}$ and $\mathrm{O} 1$ atoms move together in a direction opposite to that of Ti and O3.

The Born effective charges $Z^{*}$ are expected to be sensitive to the internal structural parameters. Figure 6 shows the evolution of the computed $Z^{*}$ for each atom. Near the cubic structure, the dependence of $Z_{\mathrm{Ti}}^{*}$ and $Z_{\mathrm{O} 3}^{*}$ on $P_{z}$ is nearly quadratic in agreement with previous calculations for $\mathrm{BaTiO}_{3} .33$ While $Z_{\mathrm{Ba}}^{*}$ and $Z_{\mathrm{O} 1}^{*}$ are rather insensitive to $P_{z}$, the Born effective charge of Ti decreases by over $30 \%$ from $P_{z}=0$ to $1.4 \mathrm{C} / \mathrm{m}^{2}$, with a corresponding increase in that of O3. More specifically, $Z_{\mathrm{Ti}}^{*}$ drops to its smallest value +4.7 while the magnitude of $Z_{\mathrm{O} 3}^{*}$ is close to its smallest value -3.9 . This structural sensitivity can be understood as being related to the anomalous values in the undistorted cubic perovskite structure, which arise from the hybridization of $\mathrm{Ti}$ and $\mathrm{O}$ orbitals in the Ti-O3 chains oriented along $\hat{z}$. As shown in Fig. 4, when $P_{z}$ increases, the Ti are displaced towards one O3 neighbor and away from the other, disrupting the chain and reducing the anemalous displacement-induced current along the chain. 34 Consequently, the magnitudes of the Born effective charges are reduced towards the nominal valences +4 for $\mathrm{Ti}$ and -2 for $\mathrm{O}$.

As the polarization and associated structural distortions become larger, the bandstructure evolves correspondingly. We find significant changes in the band structure for $P_{z}=1.8 \mathrm{C} / \mathrm{m}^{2}$ relative to that of the undistorted cubic perovskite structure. The hybridization between the Ti $3 \mathrm{~d}$ and $\mathrm{O} 2 \mathrm{p}$ bands becomes more significant, as expected from the decreased Ti-O3 distance. Some bands, such as the topmost O $2 \mathrm{p}$ band, lose the characteristic flatness that is usually seen in perovskites like $\mathrm{BaTiO}_{3} 32$ and become much more dispersive. Most importantly, the band gap decreases with increasing $P_{z}$, extrapolating to an insulator-metal transition just above 
$P_{z}=1.8 \mathrm{C} / \mathrm{m}^{2}$. The polarization is a meaningful quantity only in insulators, and therefore calculation for higher values of $P_{z}$ cannot be considered.

We carried out an analogous calculation for $\mathrm{PbTiO}_{3}$ in the cubic structure using the lattice constant deduced from experiment, $a_{0}=7.5 \mathrm{a}$ a, yielding a spontaneous polarization of $0.73 \mathrm{C} / \mathrm{m}^{2}$. The unit displacement vector as a function of $P_{z}$ is shown in the right panel of Fig. 5 , where the pattern resembles that of $\mathrm{BaTiO}_{3}$ at intermediate values of $P_{z}$. Thus, the pattern that is field-induced in $\mathrm{BaTiO}_{3}$ is characteristic of that of $\mathrm{PbTiO}_{3}$ at zero electric field. The high- $P_{z}$ regime sets in at around $1.5 \mathrm{C} / \mathrm{m}^{2}$, with the $\mathrm{Pb}$ and $\mathrm{O} 1$ atoms moving together as a pair and $\mathrm{Ti}$ and O3 moving together as a second pair.

Some general observations can be made about the effects of an electric field on the internal structural parameters. At small fields, the cations and anions move independently, following the electrostatic force corresponding to the sign of the formal valence. However, once the field-induced distortions are large enough so that short range interatomic repulsion prevents further compression of $\mathrm{Ba} / \mathrm{Pb}-\mathrm{O} 1$ and $\mathrm{Ti}-\mathrm{O} 3$ bonds, the further distortions acquire a new character in which these atoms move as pairs. From the computed values of the dynamic effective charges at $P_{z}=1.64 \mathrm{C} / \mathrm{m}^{2}$ (see Fig. 价, the Ba-O1 pair and Ti-O3 pair carry net charges very close to -2 and +2 respectively.

In summary, we have shown that the "simple" perovskite compound $\mathrm{BaTiO}_{3}$ exhibits significant nonlinearity in structure with increasing polarization, corresponding to large electric fields, while the atomic displacement pattern of $\mathrm{PbTiO}_{3}$ is relatively slowly varying. It is interesting to note that at large enough polarization, the atomic displacement pattern of $\mathrm{BaTiO}_{3}$ in fact resembles that of $\mathrm{PbTiO}_{3}$. This may help to shed some light on the factors responsible for the differences in properties between alkaline earth and $\mathrm{Pb}$-based perovskites.

\section{Non-Linear Dielectric and Piezoelectric Response}

Tunability of the dielectric and piezoelectric coefficients by an applied electric field, a property of great technological importance, is expected to be especially large in ferroelectrics due to the dependence of these coefficients on electric-field-induced structural changes such as those reported for $\mathrm{BaTiO}_{3}$ in the previous section. This behavior can be quantified by the values of the nonlinear dielectric and piezoelectric coefficients. In this section, we formulate the calculation of these nonlinear coefficients in our polarization-based framework, and give results for tetragonal $\mathrm{PbTiO}_{3}$.

The first step in this analysis is the computation of $F(\mathbf{P})$ and $\eta(\mathbf{P})$ from the minimization of $F(\mathbf{R}, \eta, \mathbf{P})$ at fixed $\mathbf{P}$. This is followed by the minimization of $F(\mathbf{P})-\mathbf{P} \cdot \mathcal{E}$ at fixed $\mathcal{E}$, directly yielding $\mathbf{P}(\mathcal{E})$ and $\eta(\mathcal{E})=\eta(\mathbf{P}(\mathcal{E}))$. From the first derivative of $\mathbf{P}(\mathcal{E})$, we obtain the field-dependent static dielectric susceptibility tensor $\chi_{\alpha \beta}(\mathcal{E})$, with the nonlinear coefficients defined through a small- $\mathcal{E}$ expansion

$$
\chi_{\alpha \beta}(\mathcal{E})=\frac{1}{\epsilon_{0}} \frac{\partial \mathbf{P}_{\alpha}(\mathcal{E})}{\partial \mathcal{E}_{\beta}}=\chi_{\alpha \beta}^{(1)}+\chi_{\alpha \beta \gamma}^{(2)} \mathcal{E}_{\gamma}+\mathcal{O}\left(\mathcal{E}^{2}\right) .
$$

The relative dielectric tensor is given by $\epsilon_{\alpha \beta}=\delta_{\alpha \beta}+$ $\chi_{\alpha \beta}$. Correspondingly, from the first derivative of $\eta(\mathcal{E})$, we obtain the field-dependent piezoelectric tensor $d_{\mu \beta}(\mathcal{E})$, with the nonlinear coefficients defined through a small- $\mathcal{E}$ expansion

$$
d_{\mu \beta}(\mathcal{E})=\frac{\partial \eta_{\mu}(\mathcal{E})}{\partial \mathcal{E}_{\beta}}=d_{\mu \beta}^{(1)}+d_{\mu \beta \gamma}^{(2)} \mathcal{E}_{\gamma}+\mathcal{O}\left(\mathcal{E}^{2}\right) .
$$

In fact, in our present implementation we perform the minimization of $F(\mathbf{R}, \eta, \mathbf{P})$ at fixed $\mathbf{P}$ in two separate steps. First, we obtain a reduced free-energy density $F(\eta, \mathbf{P})$ by minimizing with respect to $\mathbf{R}$ at fixed $\eta$ and $\mathbf{P}$. Further minimization with respect to $\eta$ to obtain $F(\mathbf{P})$ and $\eta(\mathbf{P})$ allows the computation of the zero-stress responses as in the previous paragraph. In addition, this approach allows the computation of the clamped-strain dielectric response, measured at frequencies above the resonant frequency of the sample, through minimization of $F(\eta, \mathbf{P})-\mathbf{P} \cdot \mathcal{E}$ at fixed $\mathcal{E}$ and $\eta=\eta(\mathcal{E})$, directly yielding $P(\eta(\mathcal{E}), \mathcal{E})$ and $\chi(\eta(\mathcal{E}), \mathcal{E})$. This two-step procedure is also required, as mentioned in Section IIIA, by the limitations imposed on the present implementation by the use of ABINIT 3.1. For present practical purposes, $F(\eta, \mathbf{P})$ is obtained in a parameterized form by fitting a Landau-Devonshire expansion to values of $F$ obtained by calculations for an appropriate set of $\eta$ and $\mathbf{P}$.

We have applied this procedure to compute the nonlinear dielectric and piezoelectric response of tetragonal $\mathrm{PbTiO}_{3}$ to fields along $\hat{z}$ using the $i=1$ expressions (Eq. 9). At this level of approximation, only lattice contributions to $\chi_{\alpha \beta}(\mathcal{E})$ and $d_{\mu \beta}(\mathcal{E})$ are included, and their electric-field dependence arise only through induced structural changes. However, this is expected to be a good approximation for $\mathrm{PbTiO}_{3}$, where the lattice contribution to the dielectric and piezoelectric responses dominates even at $T=0$.

With a field along $\hat{z}$, symmetry constrains the structural response to consist of a tetragonal strain, specified by two independent parameters $\eta_{1}=\eta_{x x}=\eta_{y y}$ and $\eta_{3}=$ $\eta_{z z}$, and a set of atomic displacements along the $\hat{z}$ direction described by three independent parameters. Correspondingly, we obtain an expression for $F(\mathbf{R}, \eta, \mathbf{P})=$ $F\left(\eta_{1}, \eta_{3}, P_{z}\right)$ by computing $\min _{\mathbf{R}} E(\mathbf{R}, \eta, \mathcal{E}=0)$ for a set of tetragonal cells with the constraint $\mathbf{P}\left(\mathbf{R}, \eta_{1}, \eta_{3}\right)=P_{z} \hat{z}$. The results are used to fit the parameters in a LandauDevonshire expression expanded around the minimumenergy cubic structure $\left(a_{0}=7.33\right.$ a.u. $)$,

$$
\begin{aligned}
F\left(\eta_{1}, \eta_{3}, P_{z}\right) & =E_{0}+\frac{1}{2} C_{11}\left(2 \eta_{1}^{2}+\eta_{3}^{2}\right)+C_{12}\left(2 \eta_{1} \eta_{3}+\eta_{1}^{2}\right) \\
& +A_{200} P_{z}^{2}+A_{400} P_{z}^{4}+A_{600} P_{z}^{6} \\
& +2 B_{1 y y} \eta_{1} P_{z}^{2}+B_{1 z z} \eta_{3} P_{z}^{2}
\end{aligned}
$$


TABLE I: The values of the least-squares fitted parameters in Eq. (33) at $\mathcal{E}=0$ in $\mathrm{PbTiO}_{3}$. The units are the appropriate combinations of $\mathrm{Ha}$ and $\left(\mathrm{C} / \mathrm{m}^{2}\right)^{2}$.

\begin{tabular}{cccr} 
Parameters & Values & Parameters & Values \\
\hline$E_{0}$ & -165.953 & $A_{400}$ & 0.005 \\
$C_{11}$ & 4.374 & $A_{600}$ & 0.004 \\
$C_{12}$ & 1.326 & $B_{1 z z}$ & -0.199 \\
$A_{200}$ & -0.003 & $B_{1 y y}$ & -0.049
\end{tabular}

TABLE II: Comparison of the structural parameters computed by minimizing Eq. (33) with those computed from direct 5 A calculation and those obtained from experiment 35.36

\begin{tabular}{lcccc} 
& $\Delta E(\mathrm{mHa})$ & $P_{z}^{\mathcal{E}=0}\left(\mathrm{C} / \mathrm{m}^{2}\right)$ & $a(\mathrm{Bohr})$ & $c($ Bohr $)$ \\
\hline model & 0.86 & 0.67 & 7.324 & 7.487 \\
LDA & 0.90 & 0.65 & 7.310 & 7.484 \\
$\exp$ & - & $0.75(295 \mathrm{~K})$ & 7.373 & 7.852
\end{tabular}

where $P_{z}$ is the polarization per unit volume and the truncations to sixth order in $P_{z}$ and to lowest order in the elastic and polarization-strain coupling are found to be sufficient within a standard least-squares fit. The resulting coefficients are shown in Table 1 ; statistical analysis shows that the strain coupling parameters $B_{1 y y}$ and $B_{1 z z}$ are the most sensitive to changes in the input configuration energies.

We now use this expansion to compute the field dependence of the strain and polarization under zero stress by minimizing $F\left(\eta_{1}, \eta_{3}, P_{z}\right)-\mathcal{E} \cdot P_{z}$ with respect to $\eta_{1}, \eta_{3}$ and $P_{z}$ to get $\eta_{1}(\mathcal{E}), \eta_{3}(\mathcal{E})$ and $P_{z}(\mathcal{E})$. By first considering $\mathcal{E}$ $=0$, we can confirm the validity of the parameterization by comparing the tetragonal structure obtained by minimizing the expression for $F\left(\eta_{1}, \eta_{3}, P_{z}\right)$ given by Eq. (33) with properties of the fully relaxed tetragonal groundstate structure in zero electric field. In Table III we list the energy difference $\Delta E$ between the tetragonal ground state and the cubic structure, the spontaneous polarization $P_{z}(\mathcal{E}=0)$, and the lattice parameters, finding good agreement in all respects.

Next, we consider nonzero $\mathcal{E}$. Minimizing first with respect to $\eta$ gives a free energy

$$
F\left(P_{z}\right)=A_{200} P_{z}^{2}+\widetilde{A}_{400} P_{z}^{4}+A_{600} P_{z}^{6}
$$

where

$\widetilde{A}_{400}=A_{400}+\frac{2 c_{12} B_{1 z z} B_{1 y y}-c_{11} B_{1 y y}^{2}-\frac{1}{2}\left(c_{11}+c_{12}\right) B_{1 z z}^{2}}{\left(c_{11}+2 c_{12}\right)\left(c_{11}-c_{12}\right)}$,

and $\widetilde{A}_{400}$ is found to be $4.5 \times 10^{-4} \mathrm{HaC}^{-4} \mathrm{~m}^{-8}$.

Since $A_{200}<0, F\left(P_{z}\right)$ has a double well structure, so that $F\left(P_{z}\right)-P_{z} \mathcal{E}$ has two local minima for small enough values of $\mathcal{E}$. The evolution of the two local minima with

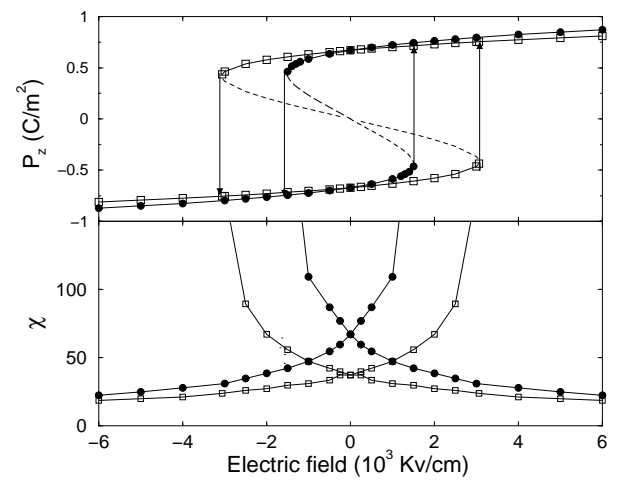

FIG. 7: Calculated polarization-vs.-electric-field hysteresis loop (upper panel) and static susceptibility $\chi(\mathcal{E})$ (lower panel) of $\mathrm{PbTiO}_{3}$ under stress-free condition (solid circle) and clamped-strain condition (open square). Dashed line corresponds to the non-accessible state (saddle point in the thermodynamic potential).

TABLE III: Comparison between theory and experiment 37 (at room temperature) for the first- and second-order dielectric constants of $\mathrm{PbTiO}_{3}$ (RT). The superscripts $\sigma$ and $\eta$ indicate whether the measurement is under constant-stress or constant-strain condition.

\begin{tabular}{lcccc} 
& $\chi_{33}^{\sigma}$ & $\chi_{33}^{\eta}$ & $\chi_{33}^{(2) \sigma}(\mathrm{nm} / \mathrm{V})$ & $\chi_{33}^{(2) \eta}(\mathrm{nm} / \mathrm{V})$ \\
\hline model & 67 & 37 & 315 & 82 \\
experiment & 79 & 33 & - & -
\end{tabular}

$\mathcal{E}$ can be summarized in the calculated hysteresis loop shown in the upper panel of Fig. 77. We find an intrinsic coercive field $\mathcal{E}_{\mathrm{c}}$ of $1.5 \mathrm{MV} / \mathrm{cm}$. From Eq. (31), we can proceed to calculate the static susceptibility $\chi_{33}(\mathcal{E})$ and the result is plotted in the lower panel of Fig. ․ Fitting this to Eq. (31), we find that the zero-field stress-free susceptibility $\chi_{33}^{(1)}$ is $\chi_{33}^{\sigma}=67$, the superscript $\sigma$ indicating stress-free conditions.

For the clamped-strain response at zero field, we fix $\eta$ at $\eta(\mathcal{E}=0)$. A different double well structure is obtained for $F^{(\eta)}\left(P_{z}\right)$, resulting in a different hysteresis loop shown in the same figure. We find an intrinsic coercive field $\mathcal{E}_{\mathrm{c}}$ of $3 \mathrm{MV} / \mathrm{cm}$. From fitting to Eq. (31), we obtain $\chi_{33}^{\eta}=37$, the superscript $\eta$ indicating the clamped-strain condition. In Table III, these values are compared with the reported experimental dielectric constants at both the constant stress and clamped-strain condition 37 which were measured below and above the sample resonant frequencies respectively. The value for $\chi_{33}^{\eta}$ can also be compared with a previous first-principles calculation. 38

In both the free-stress and fixed-strain case, the hysteresis profile of the static susceptibility shows that $\chi_{33}$ increases with field amplitude for the local minimum at $\mathcal{E}<\mathcal{E}_{\mathrm{c}}$ and decreases with increasing field for the global minimum, which is the only branch in the region above $\mathcal{E}_{\mathrm{c}}$. For each branch, we find a non-linear susceptibility $\chi_{33}^{(2) \sigma}$ of magnitude $315 \mathrm{~nm} / \mathrm{V}$ in the stress-free case. 


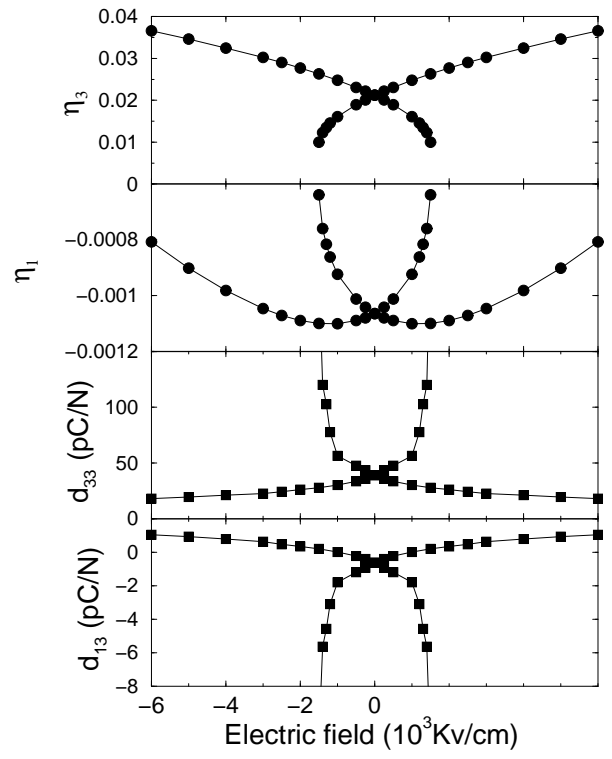

FIG. 8: The calculated equilibrium strains $\eta_{1}$ and $\eta_{3}$ and the piezoelectric tensor $d_{13}$ and $d_{33}$ as a function of the electric field in $\mathrm{PbTiO}_{3}$.

However, when the strain is clamped, the coercive field becomes larger than in the stress-free case, and the nonlinear susceptibility is more than two times smaller. In the present framework, this is not surprising since the change in the dielectric response is the result of a fieldinduced change in structure, and this change is reduced by clamping the strain. In nonzero field, the susceptibility can be either of these two values depending on whether the the system is in a single domain corresponding to the global minimum or to the local minimum, or an intermediate value if both types of domains are present.

Next, we consider the piezoelectric response (Eq. 32). In Fig. 8, we plot the equilibrium values of the strains $\eta_{1}$ and $\eta_{3}$ as a function of the electric field along the $z$ direction. The slopes of these curves give rise to the piezoelectric coefficients $d_{13}$ and $d_{33}$ which are plotted in the lower panels of the same plot. We find $d_{13}=-0.6 \mathrm{pC} / \mathrm{N}$ and $d_{33}=40 \mathrm{pC} / \mathrm{N}$, considerably less than the room temperature values measured experimentally $36(-25 \mathrm{pC} / \mathrm{N}$ and $117 \mathrm{p} / \mathrm{N}$, respectively) and computed from first principles 39 We attribute this primarily to the choice of pseudopotentials, which give a low value for the ground state tetragonal ratio $c / a$ and in particular, a value of $a$ almost unchanged from the cubic $a_{0}$. However, our calculation does serve to demonstrate the applicability of our method to the calculation of these quantities. In particular, there is to our knowledge no previous calculation of the nonlinear piezoelectric response.

\section{Field-Induced Structural Phase Transitions}

In a single crystal, the relative stability of distortedstructure phases with polarizations in different directions is expected to change as an electric field is applied. In particular, a phase transition might be induced by applying a sufficiently large field in a different direction from the polarization of the ground state. This change in phase, the result of an electric-field induced rotation of the polarization, may be accompanied by a large change in strain, manifested as a large piezoelectric response.

This "polarization rotation" mechanism was proposed in Ref. 13 to explain the experimentally observed colossal piezoelectric response to electric fields along [001] of single-crystal rhombohedral perovskite alloys such as $\left[\mathrm{Pb}\left(\mathrm{Zn}_{1 / 3} \mathrm{Nb}_{2 / 3}\right) \mathrm{O}_{3}\right]_{(1-x)}-\left[\mathrm{PbTiO}_{3}\right]_{x}(\mathrm{PZN}-\mathrm{PT})$ with compositions near the rhombohedral-tetragonal morphotropic phase boundary (R-T MPB), and has been the subject of continuing experimental 40 and theoretical 11 investigation. Particular attention has focused on the nature of the path followed by the polarization vector with increasing field strength. An effective Hamiltonian study of $\mathrm{PbZr}_{x} \mathrm{Ti}_{1-x} \mathrm{O}_{3}$ near the R-T MPB 41 showed that with increasing electric field along [111], the polarization vector of tetragonal PZT rotates continuously from the tetragonal [001] direction to the rhombehedral [111] direction through a monoclinic " $M_{A}$ " phase 42 with $\mathbf{P}$ along [uu1]. In contrast, for the case of an [001] electric field applied to rhombohedral PZT, the polarization vector does not simply follow the return path, but instead follows a discontinuous path of a kind first discussed by Noheda.40 It first rotates continuously into the $M_{A}$ phase for small field strengths, and then jumps discontinuously to a monoclinic " $M_{C}$ " phase ${ }^{22}$ with $\mathbf{P}$ along $[u 01]$ before reaching the tetragonal structure. The calculations show that a large piezoelectric response is expected for this latter type of path.

In this section, we apply the full three-dimensional formalism described in Section II to study, in a Pb-based perovskite system, the rotation of polarization by an applied electric field in the two cases most relevant to enhanced piezoelectric response near the R-T MPB: (i) application of an electric field along [111] to a tetragonal system, and (ii) application of an electric field along [001] to a rhombohedral system. For (i), we consider tetragonal $\mathrm{PbTiO}_{3}$. For (ii), we introduce a simple modification of the structural energetics of $\mathrm{PbTiO}_{3}$ to stabilize a rhombohedral ground-state structure. This follows the spirit of a view of PZN-PT and PMN-PT as large-strain $\mathrm{PbTiO}_{3}$-based systems that have been chemically "engineered" to make them marginally stable in the rhombohedral phase. 13 We do something very similar, but using a theoretical manipulation that avoids the unnecessary complexities of the real alloy systems. 


\section{Free-energy functional}

Extending the procedure described in Section IVQ to the full three-dimensional case, we first evaluate the reduced free-energy function $F(\eta, \mathbf{P})$ by minimizing $F(\mathbf{R}, \eta, \mathbf{P})$ with respect to $\mathbf{R}$ for a set of selected tetrago- nal, rhombohedral and orthorhombic structures. Strains are defined relative to the cubic structure with the experimental lattice constant ( $a_{0}=7.5$ a.u. $)$. In the range of $\eta$ and $\mathbf{P}$ of interest, we used a procedure similar to Sec. IV to fit $F(\eta, \mathbf{P})$ in a Landau-Devonshire form:

$$
\begin{aligned}
F(\eta, \mathbf{P}) & =E_{0}+C_{1}\left(\eta_{1}+\eta_{2}+\eta_{3}\right)+\frac{1}{2} C_{11}\left(\eta_{1}^{2}+\eta_{2}^{2}+\eta_{3}^{2}\right)+C_{12}\left(\eta_{2} \eta_{3}+\eta_{3} \eta_{1}+\eta_{1} \eta_{2}\right)+\frac{1}{2} C_{44}\left(\eta_{4}^{2}+\eta_{5}^{2}+\eta_{6}^{2}\right) \\
& +A_{200}\left(P_{x}^{2}+P_{y}^{2}+P_{x}^{2}\right)+A_{400}\left(P_{x}^{4}+P_{y}^{4}+P_{x}^{4}\right)+A_{220}\left(P_{y}^{2} P_{z}^{2}+P_{z}^{2} P_{x}^{2}+P_{x}^{2} P_{y}^{2}\right) \\
& +A_{600}\left(P_{x}^{6}+P_{y}^{6}+P_{z}^{6}\right)+A_{420}\left[P_{x}^{2}\left(P_{y}^{4}+P_{z}^{4}\right)+P_{y}^{2}\left(P_{z}^{4}+P_{x}^{4}\right)+P_{z}^{2}\left(P_{x}^{4}+P_{y}^{4}\right)\right]+A_{222} P_{x}^{2} P_{y}^{2} P_{z}^{2} \\
& +B_{1 x x}\left(\eta_{1} P_{x}^{2}+\eta_{2} P_{y}^{2}+\eta_{3} P_{z}^{2}\right)+B_{1 y y}\left[\eta_{1}\left(P_{y}^{2}+P_{z}^{2}\right)+\eta_{2}\left(P_{z}^{2}+P_{x}^{2}\right)+\eta_{3}\left(P_{x}^{2}+P_{y}^{2}\right)\right] \\
& +B_{4 y z}\left(\eta_{4} P_{y} P_{z}+\eta_{5} P_{z} P_{x}+\eta_{6} P_{x} P_{y}\right) .
\end{aligned}
$$

We list the least-squares fitted coefficients in the column denoted by M1 in Table IV.

Using Eq. (36), we now consider the energetics of states with different orientations of the polarization in zero field. Specifically, we consider $E(\theta, \phi, \mathcal{E}=0)$, obtained by fixing the direction of $\mathbf{P}$ along the direction specified by spherical angles $\theta$ and $\phi$, relative to the polar axis $\hat{z}$, and minimizing $F(\eta, \mathbf{P})$ with respect to the strain and the magnitude of $\mathbf{P}$. As shown in Fig. 9, the tetragonal phase $(\mathrm{T})$ with polarization along [001] is the global minimum, with a saddle point at the orthorhombic phase (O) with $\mathbf{P} \|$ [110] and a maximum at the rhombohedral phase (R), with $\mathbf{P} \|[111]$. As shown in Table $\mathbf{V}$, the structural parameters and the spontaneous polarizations agree well with the LDA results, especially for the $\mathrm{O}$ and $\mathrm{R}$ phases.

From this table, it can also be seen that the energy differences between the $\mathrm{T}, \mathrm{O}$ and $\mathrm{R}$ phases are quite small. For this reason, the parameters obtained by a global least-squares minimization do not accurately reproduce the LDA values. In particular, the energy difference between the $\mathrm{T}$ and $\mathrm{R}$ phases is seen to be much larger than

TABLE IV: Least-squares fitted values of the parameters in Eq. (36). M1, all parameters freely varied; M2, with the constraint $A_{222}=0.062 \mathrm{Ha} \mathrm{C}^{-6} \mathrm{~m}^{-12}$ (boldface). Units are the appropriate combinations of $\mathrm{Ha}$ and $\left(C / \mathrm{m}^{2}\right)^{2}$.

\begin{tabular}{c|c|c||c|r|r} 
& $\mathrm{M} 1$ & \multicolumn{1}{|c||}{$\mathrm{M} 2$} & & $\mathrm{M} 1$ & \multicolumn{1}{c}{$\mathrm{M} 2$} \\
\hline$E_{0}$ & -165.947 & -165.947 & $C_{1}$ & 0.168 & 0.168 \\
$A_{200}$ & -0.01 & -0.009 & $C_{11}$ & 3.829 & 3.973 \\
$A_{400}$ & 0.008 & 0.005 & $C_{12}$ & 1.462 & 1.484 \\
$A_{220}$ & 0.015 & -0.0007 & $C_{44}$ & 1.174 & 1.218 \\
$A_{600}$ & 0.003 & 0.004 & $B_{1 x x}$ & -0.235 & -0.234 \\
$A_{420}$ & 0.010 & 0.019 & $B_{1 y y}$ & -0.048 & -0.0525 \\
$A_{222}$ & 0.009 & $\mathbf{0 . 0 6 2}$ & $B_{4 y z}$ & -0.069 & -0.068
\end{tabular}

the LDA result. However, these features of the energy surface are crucial to the physics of the structural phase transitions. Therefore, we adjusted the fitting procedure to reproduce these relative energies accurately while using the least-squares procedure for the best overall fit to the remaining data, as follows. Rather than introduce additional parameters by including higher-order terms, we "tune" one parameter while determining the other 13 parameters by standard least-squares minimization, and choose the value for the single tuned parameter that yields the most accurate values for both the O-T and O$\mathrm{R}$ energy differences. $A_{222}$ proves to be the best choice for the tuning parameter, and with $A_{222}=0.062$ and the other parameters as given in the column denoted by M2 of Table IV, both the O-T and O-R energy differences

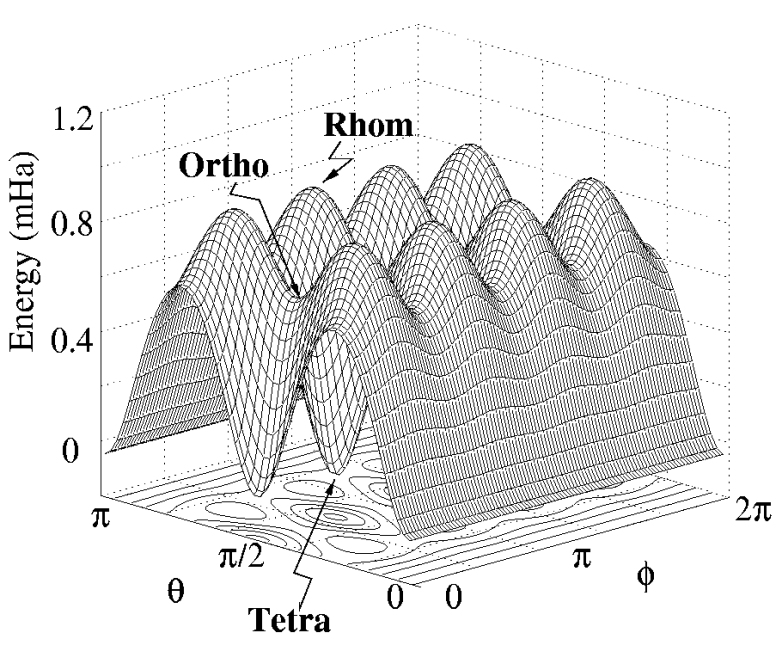

FIG. 9: Contour plot of $E(\theta, \phi, \mathcal{E}=0)$ for $\mathrm{PbTiO}_{3}$. Spherical angles $\theta$ (polar) and $\phi$ (azimuthal) indicate the direction of $\mathbf{P}$. 
TABLE V: Comparison between the structural properties of the tetragonal $(\mathrm{T})$, orthorhombic $(\mathrm{O})$, and rhombohedral $(\mathrm{R})$ phases of $\mathrm{PbTiO}_{3}$. LDA denotes direct LDA structural relaxations; M1 and M2 are as in Table IV. Units of polarization $P$, rhombohedral angle $\alpha$, cell volume $V$, and phase energies $E$ are $\mathrm{C} / \mathrm{m}^{2}$, degrees, $\mathrm{Bohr}^{3}$, and $\mathrm{mHa}$, respectively.

\begin{tabular}{c|ccc} 
& LDA & M1 & M2 \\
\hline$V_{\mathrm{T}}$ & 399.9 & 402.3 & 401.9 \\
$c / a$ & 1.024 & 1.04 & 1.03 \\
$P_{\mathrm{T}}$ & 0.65 & 0.75 & 0.71 \\
\hline$V_{R}$ & 398.4 & 397.3 & 398.7 \\
$\alpha_{\mathrm{R}}$ & 89.7 & 89.6 & 89.8 \\
$P_{\mathrm{R}}$ & 0.33 & 0.32 & 0.34 \\
\hline$V_{\mathrm{O}}$ & 398.8 & 398.1 & 399.7 \\
$\alpha_{\mathrm{O}}$ & 89.5 & 89.4 & 89.6 \\
$P_{\mathrm{O}}$ & 0.41 & 0.42 & 0.44 \\
\hline$E_{\mathrm{R}}-E_{\mathrm{O}}$ & 0.060 & 0.097 & 0.064 \\
$E_{\mathrm{O}}-E_{\mathrm{T}}$ & 0.159 & 0.639 & 0.154
\end{tabular}

as well as the structural parameters and spontaneous polarizations of all three phases are in excellent agreement with the LDA results, as shown in the last column of Table $\mathrm{V}$. Therefore, this set of parameters was used in the following calculations.

\section{Engineering a Rhombohedral Structure for $\mathrm{PbTiO}_{3}$}

In previous first-principles investigations of $\mathrm{PbTiO}_{3}$, it has been observed that the strain coupling is responsible for stabilizing the tetragonal ground state structure.44 In the simple cubic lattice, the lowest-energy structure has polarization along [111], corresponding to a rhombohedral symmetry, while the energy of the optimal state with polarization along [001] is higher. However, when the lattice is allowed to relax, the energy gain from strain coupling in the tetragonal structure is much larger than the gain in the rhombohedral structure, leading to the observed reversal of stability. In each case, the energy gain from strain coupling increases as the relevant elastic constant decreases. So, if it were possible to decrease the shear modulus $C_{44}$, there would be a critical value below which the rhombohedral state would be most stable.

Within Eq. (36), the modification of $C_{44}$ can be implemented by the inclusion of a tunable shear elastic term

$$
\widetilde{F}(\eta, \mathbf{P})=F(\eta, \mathbf{P})+\frac{1}{2} \Delta C_{44}\left(\eta_{4}^{2}+\eta_{5}^{2}+\eta_{6}^{2}\right)
$$

where $\Delta C_{44}=0$ corresponds to $\mathrm{PbTiO}_{3}$ with its natural shear elastic modulus. Using Eq. (37), we compute the zero-field energy for the optimal tetragonal, rhombohedral and orthorhombic phases as a function of $\Delta C_{44}$. This yields the phase sequence shown in Fig. 10 with the $\mathrm{T}$ and $\mathrm{R}$ phases separated by a sliver of an orthorhombic

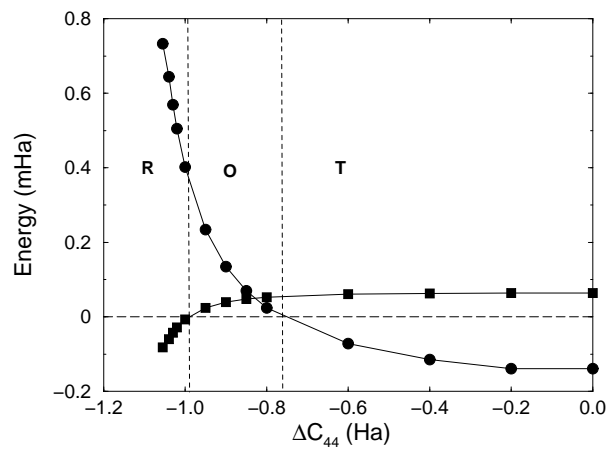

FIG. 10: The energies of the rhombohedral (square) and tetragonal (circle) phases relative to the orthorhombic phase (chosen as the zero of energy) as a function of the tunable shear modulus $\Delta C_{44}$ of $\mathrm{PbTiO}_{3}$, calculated using Eq. (37). The ranges of $\Delta C_{44}$ in which the tetragonal, orthorhombic and rhombohedral phases are most stable are separated by vertical dashed lines and indicated by $\mathrm{T}, \mathrm{O}$ and $\mathrm{R}$ respectively.

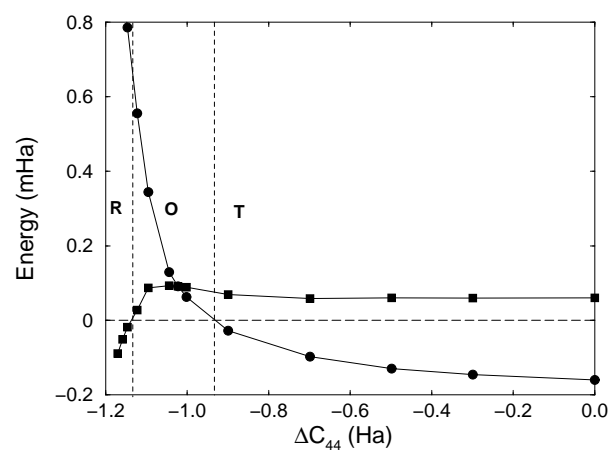

FIG. 11: Same as Fig. 10 but calculated using a direct LDA approach. As in Fig. 10, an orthorhombic window appears, though the phase boundaries are slightly shifted.

phase. This phase sequence is very remipiscent of that of the $\mathrm{Pb}\left(\mathrm{Zn}_{1 / 3} \mathrm{Nb}_{2 / 3}\right) \mathrm{O}_{3}-\mathrm{PbTiO}_{3}$ system 12 with the tunable parameter being the proportion of $\mathrm{PbTiO}_{3}$. The stability of the orthorhombic phase reflects the importance of the sixth-order terms in Eq. (36), as in a fourth-order model only tetragonal and rhombohedral structures are possible minima.

To check that the observed phase sequence is not an artifact of our fit, we have computed the structural parameters and energies of the tetragonal, orthorhombic and rhombohedral phases as a function of $\Delta C_{44}$ through direct LDA calculations. For consistency with Eq. (37), we implement the adjustment of the shear modulus as an additional applied stress

$$
\Delta \sigma_{i}=-\Delta C_{44} \eta_{i}
$$

where $\sigma_{i}$ (with $i=4,5,6$ ) are the shear stress components in Voigt notation. The results, given in Fig. 11, show the same T-O-R phase sequence as Fig. 10. While the T-O and O-R phase boundaries are slightly shifted, the width of the orthorhombic window is comparable to 

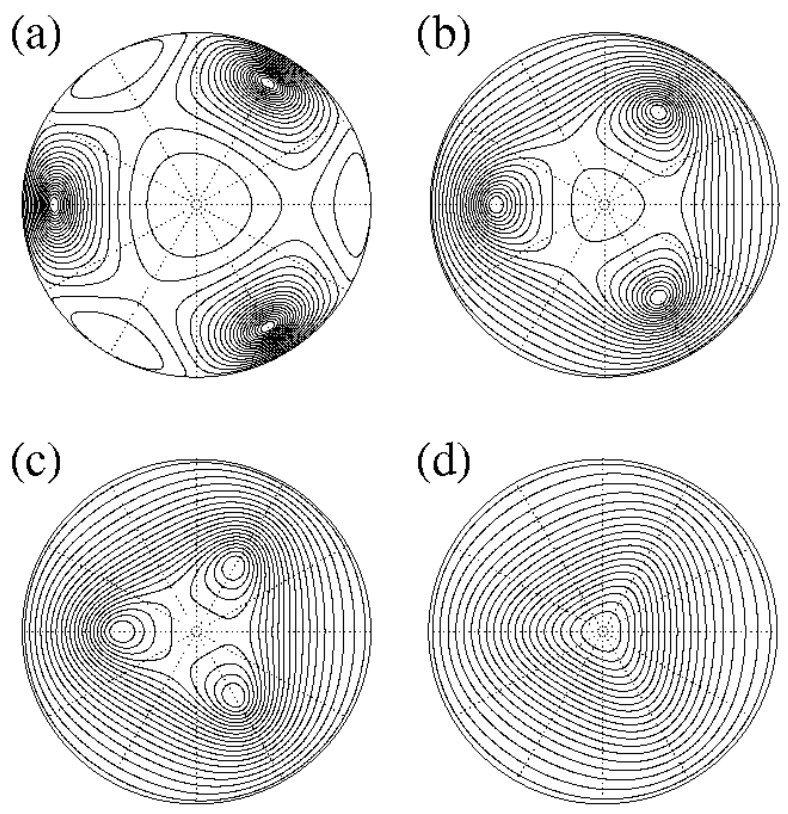

FIG. 12: Contour maps of $E(\theta, \phi, \mathcal{E})$ on the upper hemisphere $0 \leq \theta \leq \frac{1}{2} \pi$ for an electric field of magnitude $\mathcal{E}$ applied along the pseudocubic [111] direction to tetragonal $\mathrm{PbTiO}_{3}$. The contour is equally spaced in $\log \left(E-E_{\min }+\delta\right)$, where $E_{\min }$ is the global minimum and $\delta$ is a small offset. The central axis points along the [111] direction. (a)-(d) correspond to electric fields of $0,0.86,1.73$, and $3.46 \times 10^{3} \mathrm{kV} / \mathrm{cm}$, respectively.

that in Fig. 10. Thus, in the following, using Eq. (37) with a particular value of $\Delta C_{44}$, we expect results which would reflect a direct LDA calculation, though perhaps with a slightly different $\Delta C_{44}$.

\section{Electric-field-induced phase transitions}

In a single crystal, the relative stability of phases with polarizations in different directions is expected to change as an electric field is applied. In particular, a phase transition might be induced by applying a sufficiently large field in a different direction from the polarization of the ground state. Here, we consider two such cases: tetragonal $\mathrm{PbTiO}_{3}$ in an electric field along [111], and rhombohedral " $\mathrm{PbTiO}_{3}$," stabilized by a nonzero value of $\Delta C_{44}$, in an electric field along [001].

First, we consider tetragonal $\mathrm{PbTiO}_{3}\left(\Delta C_{44}=0\right)$ in an electric field along [111], which tends to favor a rhombohedral direction for the polarization. To investigate the evolution of various phases with $\mathcal{E}_{111}$, where $\mathcal{E}_{111}$ is the magnitude of the electric field, we perform the minimization in two steps. First, we transform the Euclidean coordinates $\left(P_{x}, P_{y}, P_{z}\right)$ into spherical coordinates $(P, \theta, \phi)$ and compute

$$
E\left(\theta, \phi, \mathcal{E}_{111}\right)=\min _{P, \eta}\left[F(\eta, \mathbf{P})-\mathcal{E}_{111}\left(P_{x}+P_{y}+P_{z}\right) / \sqrt{3}\right]
$$

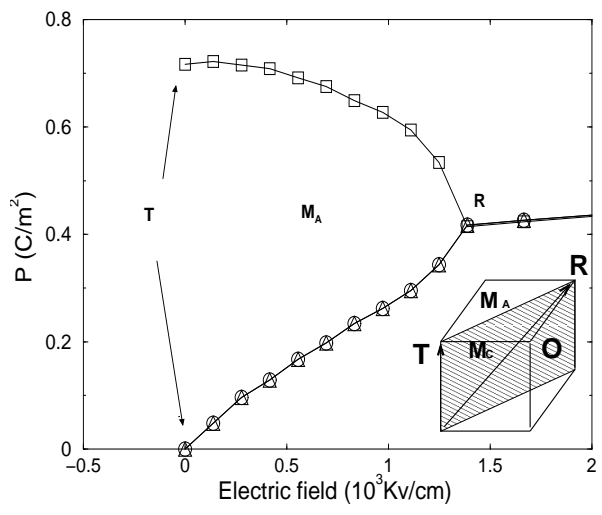

FIG. 13: The Cartesian component $P_{x}$ (circle), $P_{y}$ (triangle), $P_{z}$ (square) of the polarization as a function of the magnitude of the electric field applied along the [111] pseudocubic direction in $\mathrm{PbTiO}_{3}$. Inset shows the polarization path.

Then, we locate the minima on the sphere of polarization directions parametrized by $\theta$ and $\phi$.

The evolution of the phase stability can be readily displayed by the contour plots of $E\left(\theta, \phi, \mathcal{E}_{111}\right)$ shown in Fig. 12. At zero electric field, the tetragonal structure appears as a three-fold degenerate energy minimum in the hemisphere shown. As $\mathcal{E}_{111}$ increases, the minima migrate from the tetragonal positions along the lines corresponding to the monoclinic $M_{A}$ phase (three-fold degenerate) and eventually reach the rhombohedral point at the center of the hemisphere.

Figure 13 shows how the polarization components of tetragonal $\mathrm{PbTiO}_{3}$ evolve with the amplitude of $\mathcal{E}_{111}$. At $\mathcal{E}_{111}=0$, the only non-zero component is $P_{z}$. As $\mathcal{E}$ increases, $P_{x}=P_{y}$ grow while $P_{z}$ slowly decreases. The structure thus enters the $M_{A}$ monoclinic phase. When $\mathcal{E}$ reaches $1.4 \times 10^{3} \mathrm{kV} / \mathrm{cm}$, the three components merge and the system enters the rhombohedral phase where the polarization vector points along the pseudocubic [111] direction. While rotating, the polarization vector remains in the (110) plane, as shown in the inset of Fig. 13.

Next, we consider rhombohedral "PbTiO 3 " with $\Delta C_{44}=-1.1 \mathrm{Ha}$ (see Fig. 10) in an electric field along the [001] direction, which tends to favor a tetragonal direction for the polarization. The analogue of Eq.( 39) is

$$
E\left(\theta, \phi, \mathcal{E}_{001}\right)=\min _{P, \eta}\left[\widetilde{F}(\eta, \mathbf{P})-\mathcal{E}_{001} P_{z}\right]
$$

The energy contour plot in this case is shown in Fig. 14 . In zero electric field, the system is in a rhombohedral phase with an eight-fold degenerate minimum. For small nonzero $\mathcal{E}_{001}$, the energy minima correspond to a $M_{A}$ phase as shown in Fig. 14(b-c) where there are four degenerate minima lying in the (110) plane. At a critical value of $\mathcal{E}_{001}$, the energy minima jump to four-fold points in the (100) plane, as can be seen in Fig. 14(d). The four minima then move smoothly towards the [001] axis, finally merging to yield the tetragonal phase. 

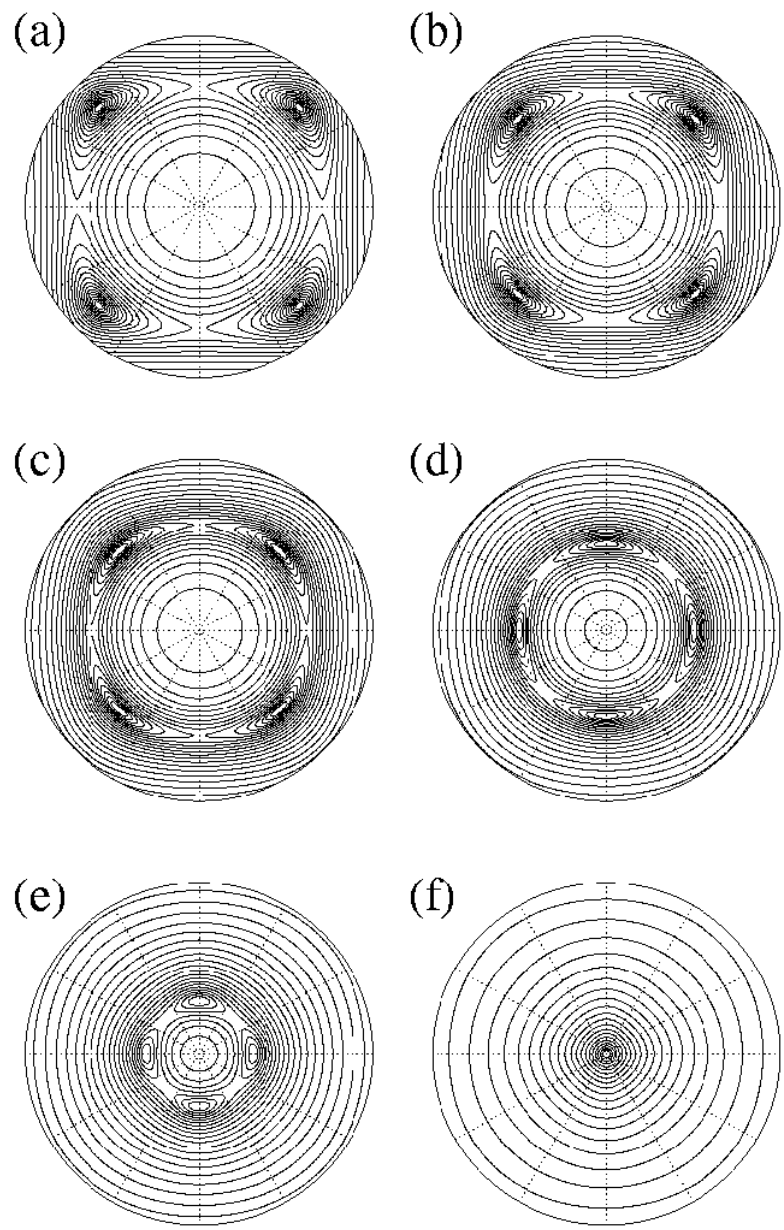

FIG. 14: Contour map of the free energy (upper hemisphere) when an electric field is applied to rhombohedral " $\mathrm{PbTiO}_{3}$ " $\left(\Delta C_{44}=-1.1 \mathrm{Ha}\right)$ along the pseudocubic [001] direction. The central axis corresponds to one of the tetragonal directions. (a)-(f) correspond to electric field magnitudes of $0,1.4,2.8$, 7,14 , and $19 \times 10^{3} \mathrm{kV} / \mathrm{cm}$, respectively.

Figure 15 shows how the polarization components of rhombohedral " $\mathrm{PbTiO}_{3}$ " evolve with the amplitude of $\mathcal{E}_{001}$. Under zero applied electric field, the polarization vector starts along the pseudocubic [111] direction $\left(P_{x}=\right.$ $\left.P_{y}=P_{z}=0.56 \mathrm{C} / \mathrm{m}^{2}\right)$. As $\mathcal{E}_{001}$ increases, the structure enters an $M_{A}$ phase in which $P_{x}$ and $P_{y}$ remain equal, but become less than $P_{z}$. $P_{x}$ and $P_{y}$ keep dropping until $P_{y}$ shows a sudden jump to zero at around $4.5 \times 10^{3} \mathrm{kV} / \mathrm{cm}$. At the same time, both $P_{x}$ and $P_{z}$ exhibit an upward jump in their values. The new phase corresponds to a different monoclinic phase denoted by $M_{C}$. The structure remains in the $M_{C}$ phase until $P_{x}$ also drops to zero at around $19 \times 10^{3} \mathrm{kV} / \mathrm{cm}$, yielding a tetragonal phase. As the field increases further, $P_{z}$ continues to increase smoothly.

In this section, we have seen that a small modification of the structural energetics of $\mathrm{PbTiO}_{3}$ can yield a complex polarization path quite similar to that proposed by Nohedat 40 and observed in simulations of PZT.41 Addi-

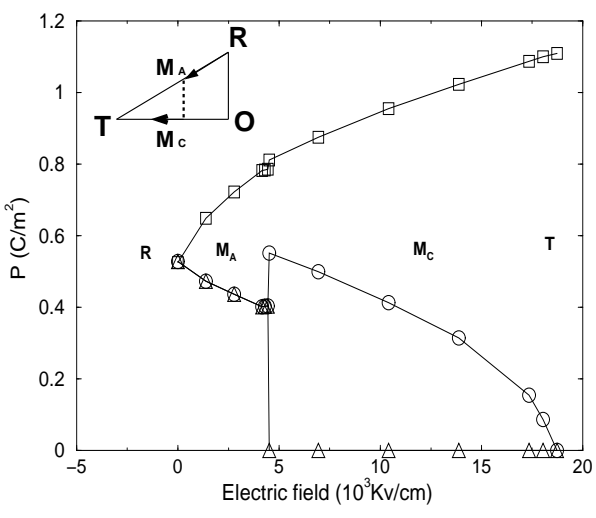

FIG. 15: Same as Fig. 13, for an electric field applied along the [001] pseudocubic direction in rhombohedral " $\mathrm{PbTiO}_{3}$ " obtained with $\Delta C_{44}=-1.1 \mathrm{Ha}$.

tional calculations, for example of the lattice parameters as a function of electric field, may assist in achieving a direct experimental observation of this behavior. In addition, further exploration within this framework may suggest ways to produce and control complicated polarization paths in real systems.

\section{SUMMARY}

In this paper, we have introduced a formalism for computing the structural response of an insulating system to a static homogeneous macroscopic electric field. We have shown that, in the presence of an electric field, the thermodynamic potential $E(\mathbf{R}, \eta, \mathcal{E})$ can be minimized by introducing a related thermodynamic potential $F(\mathbf{R}, \eta, \mathbf{P})$ in which the polarization $\mathbf{P}$ is treated as a fundamental variable. Corresponding to each polarization $\mathbf{P}$, the equilibrium values for the internal coordinates $\mathbf{R}$ and $\eta$ as well as the minimum of this energy functional can be computed. Consequently, one arrives at an energy functional that only depends on $\mathbf{P}$ and where the effect of a homogeneous electric field can be treated exactly by adding a linear term $-\mathcal{E} \cdot \mathbf{P}$ to this functional.

In practice, when $E(\mathbf{R}, \eta, \mathcal{E})$ is expanded to first order in $\mathcal{E}$, the minimization is reduced to one over the internal coordinates constrained by a fixed polarization computed at zero electric field. We have implemented a minimization scheme in the framework of a modified DFPT, using a consistent discretization formula that was developed for the response to an electric field. Consequently, the computed response is compatible with the Berry-phase polarization, which is a central quantity in the formalism.

It is important to note that the present $i=1$ theory is most useful for systems in which the response to an electric field is dominated by the changes in atomic coordinates and strains rather than by electronic polarization. Ferroelectric and nearly-ferroelectric materials are among the best examples. We therefore look forward to 
future applications of our new approach for a variety of purposes, for example, ferroelectric alloys and ferroelectric superlattices. Applying the method to the so called "high-K materials" to study their dielectric properties in the presence of an applied electric field also appears to be a promising direction.

Though the higher-order (say $i=2$ ) theory requires higher $(\geq 3)$ order energy derivatives, this does not preclude its application. As mentioned in Appendix A, it is possible to approximate certain response quantities that are related to the third derivatives by constant values from a single structure, if they show only small variations within the range of the polarization studies. Systems that may satisfy such a condition will be the subject of further investigation.

\section{Acknowledgments}

This work was supported by ONR Grants N0014-971-0048 and N00014-00-1-0261. The work of K.M.R. was performed in part at the Aspen Center for Physics. We would like to thank X. Gonze for his interest in the work and valuable discussions on the Abinit code. We acknowledge Ph. Ghosez and M. Veithen for their help on the FHI pseudopotentials.

\section{APPENDIX A: SECOND-ORDER EXPANSION FORMALISM}

This appendix presents the formalism in Section II for truncation of the sum in Eq. (6) at $i=2$, that is, at second order in the electric field $\mathcal{E}$. At this order, the thermodynamic potential $E(\mathbf{R}, \eta, \mathcal{E})$ is replaced by $E_{2}(\mathbf{R}, \eta, \mathcal{E})$, which is the sum of the first three terms in Eq. (6).

We recall the definition of the dielectric susceptibility tensor

$$
\chi_{\alpha \beta}(\mathbf{R}, \eta, \mathcal{E})=-\frac{1}{\epsilon_{0}} \frac{\partial^{2} E(\mathbf{R}, \eta, \mathcal{E})}{\partial \mathcal{E}_{\alpha} \partial \mathcal{E}_{\beta}}=\frac{1}{\epsilon_{0}} \frac{\partial P_{\alpha}(\mathbf{R}, \eta, \mathcal{E})}{\partial \mathcal{E}_{\beta}}
$$

Therefore, we can write

$$
E_{2}(\mathbf{R}, \eta, \mathcal{E})=E(\mathbf{R}, \eta, \mathcal{E})-\sum_{\alpha} P_{\alpha}(\mathbf{R}, \eta, 0) \mathcal{E}_{\alpha}-\frac{\epsilon_{0}}{2} \sum_{\alpha \beta} \mathcal{E}_{\alpha} \mathcal{E}_{\beta} \chi_{\alpha \beta}(\mathbf{R}, \eta, 0)
$$

and

$$
P_{2, \alpha}(\mathbf{R}, \eta, \mathcal{E})=P_{\alpha}(\mathbf{R}, \eta, 0)+\epsilon_{0} \sum_{\beta} \mathcal{E}_{\beta} \chi_{\alpha \beta}(\mathbf{R}, \eta, 0) .
$$

The computation of $F(\mathbf{P})$ (Eq. 2) for a given $\mathbf{P}$ proceeds by the minimization of $E(\mathbf{R}, \eta, \lambda)+\lambda \cdot \mathbf{P}$ following the procedure in Section IIIA. This involves computing the derivatives

$$
\begin{gathered}
\frac{\partial E_{2}(\mathbf{R}, \eta, \lambda)}{\partial R_{i \gamma}}=\frac{\partial E(\mathbf{R}, \eta, 0)}{\partial R_{i \gamma}}-\sum_{\alpha} \frac{\partial P_{\alpha}(\mathbf{R}, \eta, 0)}{\partial R_{i \gamma}} \lambda_{\alpha}-\frac{\epsilon_{0}}{2} \sum_{\alpha \beta} \lambda_{\alpha} \lambda_{\beta} \frac{\partial \chi_{\alpha \beta}(\mathbf{R}, \eta, 0)}{\partial R_{i \gamma}} \\
\frac{\partial E_{2}(\mathbf{R}, \eta, \lambda)}{\partial \eta_{\mu}}=\frac{\partial E(\mathbf{R}, \eta, 0)}{\partial \eta_{\mu}}-\sum_{\alpha} \frac{\partial P_{\alpha}(\mathbf{R}, \eta, 0)}{\partial \eta_{\mu}} \lambda_{\alpha}-\frac{\epsilon_{0}}{2} \sum_{\alpha \beta} \lambda_{\alpha} \lambda_{\beta} \frac{\partial \chi_{\alpha \beta}(\mathbf{R}, \eta, 0)}{\partial \eta_{\mu}} \\
\frac{\partial E_{2}(\mathbf{R}, \eta, \lambda)}{\partial \lambda_{\alpha}}=-P_{\alpha}(\mathbf{R}, \eta, 0)-\epsilon_{0} \sum_{\beta} \chi_{\alpha \beta}(\mathbf{R}, \eta, 0) \lambda_{\beta}+P_{\alpha} .
\end{gathered}
$$

These are related to the corresponding derivatives in the $i=1$ case (Eq. 10) by the addition of terms one order higher in $\lambda$. From Eq. (A6), we see that at this order $\mathbf{P}(\mathbf{R}, \eta, \lambda)$ includes an electronic contribution $\epsilon_{0} \sum_{\beta} \chi_{\alpha \beta}(\mathbf{R}, \eta, 0) \lambda_{\beta}$. The effective forces and stresses (Eqs. A4 and A5) involve the derivatives of $\chi$ with re- spect to $\mathbf{R}$ and $\eta$. While these are in principle obtainable from the $2 n+1$ theorem, they are not routinely calculated in current DFPT codes. For cases where the lattice contribution to $\mathbf{P}$ dominates, it is reasonable however to approximate the $\mathbf{R}$ and $\eta$ dependence of $\chi$ by evaluating it at the zero-field equilibrium structure. A more accurate but still practical approximation would include the first 
order changes with respect to $\delta \mathbf{R}$ and $\delta \eta$, with the derivatives computed through a finite difference approach.

\section{APPENDIX B: MULTIBAND DISCRETIZATION FORMULA}

In Sec. IIIB we presented a finite-difference formula, Eq. (30), representing the derivative $i \partial\left|u_{k}\right\rangle / \partial k$ in the single-band 1D case. In this Appendix we generalize the derivation in order to obtain a corresponding formula for the multiband 3D case.

The general expression for the electronic polarization in $3 \mathrm{D}$ is easily reduced to a sum of $1 \mathrm{D}$ Berry phases over strings of $\mathbf{k}$ points 16 We can write

$$
\mathbf{P}=\frac{1}{V N_{k}} \sum_{k_{\perp}} \sum_{\alpha} \mathbf{R}_{\alpha} P_{\alpha}\left(k_{\perp}\right)
$$

where $V$ is the cell volume, $\alpha$ labels the three primitive real-space lattice vectors $\mathbf{R}_{\alpha}$ conjugate to the primitive reciprocal-space vectors $\mathbf{G}_{\alpha}$, and $k_{\perp}$ runs over a $2 \mathrm{D}$ mesh of $N_{k}$ positions in the reciprocal-space directions perpendicular to $\alpha$. The contribution from the string $\mathcal{S}\left(k_{\perp}\right)$ of k-points running parallel to $\mathbf{G}_{\alpha}$ at a given $k_{\perp}$ is

$$
P_{\alpha}\left(k_{\perp}\right)=-\frac{f e}{2 \pi} \sum_{\mathbf{k} \in S\left(k_{\perp}\right)} \operatorname{Im} \ln \operatorname{det} M^{(\mathbf{k}, \mathbf{k}+\mathbf{b})}
$$

where $f=2$ for spin,

$$
M_{m n}^{(\mathbf{k}, \mathbf{k}+\mathbf{b})}=\left\langle u_{m \mathbf{k}} \mid u_{n, \mathbf{k}+\mathbf{b}}\right\rangle
$$

is the overlap matrix formed of inner products between Bloch orbitals on neighboring $\mathbf{k}$-points on the string, $\mathbf{b}$ is the separation between neighboring points on the string, and $m$ and $n$ run over the occupied valence bands. Equation (B2) is essentially the multi-band generalization of Eq. (28) of Sec. III B.

For the remainder of this Appendix, we drop the 3D notation and start from the $1 \mathrm{D}$ version

$$
P=-\frac{f e}{2 \pi} \sum_{k} \operatorname{Im} \ln \operatorname{det} M^{(k, k+b)}
$$

of Eq. (B2), and correspondingly for Eq. (B3). Our task is to compute the variation $\delta P$ arising from the first-order variations of the wavefunctions in Eq. (B4). Focusing on a single wavevector $k$ and its neighbor $k^{\prime}=k+b$ and letting $M=M^{\left(k, k^{\prime}\right)}$, our central task is clearly to compute the first-order variation of the phase

$$
\phi=\operatorname{Im} \ln \operatorname{det} M \text {. }
$$

Using

$$
\operatorname{det} M=\sum_{\hat{\mathrm{p}}}(-1)^{\hat{\mathrm{p}}} \prod_{n}\left\langle u_{n k} \mid u_{\hat{\mathrm{p}}(n) k^{\prime}}\right\rangle
$$

where $\hat{p}$ runs over all possible permutations among the occupied bands, the change in this phase from a firstorder change in the wavefunctions at $k$ is

$$
\delta \phi=\operatorname{Im} \frac{\delta \operatorname{det} M}{\operatorname{det} M_{0}}
$$

where

$$
\begin{aligned}
\delta \operatorname{det} M & =\sum_{\hat{\mathrm{p}}}(-1)^{\hat{\mathrm{p}}} \sum_{n}\left\langle\delta u_{n k} \mid u_{\hat{\mathrm{p}}(n) k^{\prime}}\right\rangle \prod_{m \neq n}\left\langle u_{m k} \mid u_{\hat{\mathrm{p}}(m) k^{\prime}}\right\rangle \\
& =\sum_{\hat{\mathrm{p}}}(-1)^{\hat{\mathrm{p}}} \sum_{n}\left\langle\delta u_{n k} \mid u_{\hat{\mathrm{p}}(n) k^{\prime}}\right\rangle \prod_{m \neq n} M_{0, m \hat{\mathrm{p}}(m)}(\mathrm{B} 8)
\end{aligned}
$$

Here $M_{0}$ is the matrix $M$ evaluated before variation of the wavefunctions.

Unfortunately, Eq. (B8) does not lend itself to simple evaluation. However, we can reduce Eq. (B8) to a trivial form as follows. Consider a linear transformation

$$
\left|\tilde{u}_{n k^{\prime}}\right\rangle \equiv \sum_{m} A_{m n}\left|u_{m k^{\prime}}\right\rangle
$$

among the occupied states at $k^{\prime}$, where $A$ is a nonsingular (but not necessarily unitary) matrix. Letting $\widetilde{M}_{m n}=\left\langle u_{m k} \mid \tilde{u}_{n k^{\prime}}\right\rangle$, it follows that $\widetilde{M}=M A$ and thus $\operatorname{det}(\widetilde{M})=\operatorname{det}(M) \operatorname{det}(A)$. Since $A$ is a constant matrix,

$$
\delta \ln \operatorname{det} \widetilde{M}=\delta \ln \operatorname{det} M .
$$

We thus have the freedom to evaluate Eqs. (B7) and (B8) with the substitutions $M \rightarrow \widetilde{M}, M \rightarrow \widetilde{M}_{0}$ and $u_{m k^{\prime}} \rightarrow$ $\tilde{u}_{m k^{\prime}}$, where $\widetilde{M}=M A$ and $\widetilde{M}_{0}=M_{0} A$, for arbitrary $A$.

The obvious choice is $A=M_{0}^{-1}$. We then find that the only permutation that survives in Eq. (B8) is the identity and the denominator of Eq. (B7) becomes unity, so that

$$
\delta \phi=\operatorname{Im} \sum_{n}\left\langle\delta u_{n k} \mid \tilde{u}_{n k^{\prime}}\right\rangle
$$

where

$$
\left|\tilde{u}_{n k^{\prime}}\right\rangle=\sum_{m}\left(M_{0}^{-1}\right)_{m n}\left|u_{m k^{\prime}}\right\rangle
$$

Eq. (B11) can also be written neatly as

$$
\delta \phi=\operatorname{Im} \operatorname{Tr}\left(\delta M \cdot M_{0}^{-1}\right) .
$$

Carrying out similar manipulations for the connection between $k$ and $k-b$, we can define

$$
\left|v_{n k}\right\rangle \equiv \frac{i}{2 b}\left(\left|\tilde{u}_{n, k+b}\right\rangle-\left|\tilde{u}_{n, k-b}\right\rangle\right)
$$

which becomes the finite-difference representation of $i \partial\left|u_{n k}\right\rangle / \partial k$ in the multiband case, analogous to Eq. (30). It is easy to check the orthogonality of the $v_{n k}$ to the occupied subspace,

$$
\left\langle u_{n k} \mid v_{m k}\right\rangle=\frac{i}{2 b}\left(\delta_{n m}-\delta_{n m}\right)=0,
$$


thus removing the need for explicit application of a conduction-band projector onto the $\left|v_{n k}\right\rangle$ when computing the right-hand side of Eq. (25). Since $\left\langle u_{m k} \mid \tilde{u}_{n k^{\prime}}\right\rangle=$ $\delta_{m n}$, we can think of $\left|\tilde{u}_{n k^{\prime}}\right\rangle$ defined in Eq. (B12) as a phase-aligned and amplitude-corrected "partner" to $\left|u_{n k}\right\rangle$ formed from the occupied subspace at $k^{\prime}$, and $\left|v_{n k}\right\rangle$ is proportional to the difference between the "partners" at $k+b$ and $k-b$.
Finally, the variation of Eq. (B4) becomes

$$
\delta P=\frac{f e b}{\pi} \sum_{k} \operatorname{Re}\left\langle\delta u_{n k} \mid v_{n k}\right\rangle
$$

in analogy with Eq. (29).

Our implementation of this scheme into ABINIT is based on Eqs. (B11. B16) above.
1 R. W. Nunes and D. Vanderbilt, Phys. Rev. Lett 73, 712 (1994).

2 R. W. Nunes and X. Gonze, Phys. Rev. B 63, 155107 (2001).

3 S. Baroni, P. Giannozzi and A. Testa, Phys. Rev. Lett, 58, 1861 (1987). P. Giannozzi, S. de Gironcoli, P. Pavone, and S. Baroni, Phys. Rev. B 43, 7231 (1991).

4 X. Gonze and J. P. Vigneron, Phys. Rev. B 39, 13120 (1989).

5 X. Gonze, Phys. Rev. A 52, 1096 (1995).

6 P. Fernández, A. Dal Corso, A. Baldereschi, and F. Mauri, Phys. Rev. B 55, R1909 (1997).

7 X. Gonze, Ph. Ghosez and R. W. Godby, Phys. Rev. Lett. 74, 4035 (1995).

8 R. Resta, Phys. Rev. Lett. 77, 2265 (1996).

9 R. M. Martin and G. Ortiz, Phys. Rev. B 56, 1124 (1997).

10 X. Gonze, Ph. Ghosez and R. W. Godby, Phys. Rev. Lett. 78, 294 (1997).

11 N. Sai, B. Meyer and D. Vanderbilt, Phys. Rev. Lett. 84, 5636 (2000); and Fundamental Physics of Ferroelectrics 2001, H. Krakauer, ed. (AIP, Melville, New York, 2001).

12 D. E. Cox. B. Noheda and G. Shirane, Y. Uesu, K. Fujishiro and Y. Yamada, Appl. Phys. Lett. 79, 400 (2001); cond-mat/010864.

13 H. Fu and R. E. Cohen, Nature 403, 281 (2000).

14 S. Froyen and M. L. Cohen, J. Phys. C 19, 2623 (1986).

15 A. F. Devonshire, Phil. Mag. 40, 1040 (1949); ibid. 42, 1065 (1951); Adv. Phys. 3, 85 (1954).

16 R. D. King-Smith and D. Vanderbilt, Phys. Rev. B 47, 1651 (1993).

17 X. Gonze and C. Lee, Phys. Rev. B 55, 10355 (1997).

18 For a separable potential, see Ref.17.

19 X. Gonze, Phys. Rev. B 55,10337 (1997).

20 R. Pick, M. H. Cohen and R. M. Martin Phys. Rev. B 1, 910 (1970).

21 The ABINIT code is a collaborative project of the Université Catholique de Louvain, Corning Inc., and other contributors (URL http://www.pcpm.ucl.ac.be/abinit).

22 M. Fuchs, M. Scheffler, Comput. Phys. Commun. 119, 67 (1999).
23 N. Troullier, J. L. Martins, Phys. Rev. B 43, 1993 (1991).

24 D. Ceperley, Phys. Rev. B 18, 3126, (1978); D. M. Ceperley and B. J. Alder, Phys. Rev. Lett 45, 566 (1980).

25 J.P. Perdew and Y. Wang, Phys. Rev. B 45, 13244 (1992).

26 M. Teter, Phys. Rev. B 48, 5031 (1993).

27 J. P. Perdew and A. Zunger, Phys., Rev. B 23, 5048 (1981).

28 M. E. Lines and A. M. Glass, Physics of Ferroelectrics and Related Materials, (Clarendon Press, Oxford, 1977).

29 Landolt-Bornstein Numerical Data and Functional Relationships in Science and Technology, edited by T. Mitsui et al. (Springer-Verlag, Berlin, 1981).

${ }^{30}$ R. E. Cohen and H. Krakauer, Ferroelectrics 136, 65 (1992).

31 R. E. Cohen, Nature 258, 136 (1992).

32 R. D. King-Smith and D. Vanderbilt, Phys. Rev. B 49, 5828 (1994).

33 Ph. Ghosez, J.-P. Michenaud, X. Gonze, Phys. Rev. B 58, 6224, (1998).

${ }^{34}$ W. Zhong, D. Vanderbilt, R. D. King-Smith and K. M. Rabe, Ferroelectrics 164, 291 (1995).

35 V. G. Gavrilyachenko, R. I. Spinko, M. A. Martynenko, E. G. Fesenko, Fiz. Tverd. Tela 12,1532 (1970) [Sov. Phys. -Solid State 12, 1203 (1970)].

36 M. J. Haun, E. Fruman, S. J. Jang, S. A. McKinstry and L.E. Cross, J. Appl. Phys. 62, 3331 (1987).

37 Z. Li, M. Grimsditch, G. M. Foster and S.-K. Chan, J. Phys. Chem, Sol. 57, 1433 (1996).

38 U. V. Waghmare, unpublished.

39 First-Principles Calculations for Ferroelectrics, Fifth Williamsburg Workshop, R.E. Cohen, ed. (AIP, Woodbury, New York, 1998),pp.61

40 B. Noheda, D. E. Cox, G. Shirane, S. E. Park, L. E. Cross and Z. Zhong, Phys. Rev. Lett 86, 3891 (2001).

41 L. Bellaiche, A. García and D. Vanderbilt, Phys. Rev. B 64, 060103 (2001).

42 D. Vanderbilt and M. H. Cohen, Phys. Rev. B. 63, 094108 (2001).

43 R. E. Cohen, J. Phys. Chem. Solids 61, 139, (2000).

44 R. E. Cohen, H. Krakauer, Phys. Rev. B 42, 6416 (1990). 